\title{
A fluid-mechanics based classification scheme for surface transient storage in riverine environments: quantitatively separating surface from hyporheic transient storage
}

\author{
T. R. Jackson ${ }^{1}$, R. Haggerty ${ }^{2}$, and S. V. Apte $^{3}$ \\ ${ }^{1}$ Water Resources Engineering Program and College of Earth, Ocean, and Atmospheric Sciences, Oregon State University, \\ 104 CEOAS Admin. Bldg., Corvallis, OR 97331-5503, USA \\ ${ }^{2}$ College of Earth, Ocean, and Atmospheric Sciences and Institute for Water and Watersheds, Oregon State University, \\ 104 CEOAS Admin. Bldg., Corvallis, OR 97331-5503, USA \\ ${ }^{3}$ School of Mechanical, Industrial, and Manufacturing Engineering, Oregon State University, 204 Rogers Hall, \\ Corvallis, OR 97331-6001, USA \\ Correspondence to: T. R. Jackson (jacksotr@engr.orst.edu), R. Haggerty (haggertr@geo.oregonstate.edu), \\ and S. V. Apte (sva@engr.orst.edu)
}

Received: 16 February 2013 - Published in Hydrol. Earth Syst. Sci. Discuss.: 4 April 2013

Revised: 14 June 2013 - Accepted: 17 June 2013 - Published: 15 July 2013

\begin{abstract}
Surface transient storage (STS) and hyporheic transient storage (HTS) have functional significance in stream ecology and hydrology. Currently, tracer techniques couple STS and HTS effects on stream nutrient cycling; however, STS resides in localized areas of the surface stream and HTS resides in the hyporheic zone. These contrasting environments result in different storage and exchange mechanisms with the surface stream, which can yield contrasting results when comparing transient storage effects among morphologically diverse streams. We propose a fluid mechanics approach to quantitatively separate STS from HTS that involves classifying and studying different types of STS. As a starting point, a classification scheme is needed. This paper introduces a classification scheme that categorizes different STS in riverine systems based on their flow structure. Eight STS types are identified and some are subcategorized based on characteristic mean flow structure: (1) lateral cavities (emergent and submerged); (2) protruding in-channel flow obstructions (backward- and forward-facing step); (3) isolated in-channel flow obstructions (emergent and submerged); (4) cascades and riffles; (5) aquatic vegetation (emergent and submerged); (6) pools (vertically submerged cavity, closed cavity, and recirculating reservoir); (7) meander bends; and (8) confluence of streams. The long-term goal is to use the classification scheme presented to develop
\end{abstract}

predictive mean residence times for different STS using fieldmeasurable hydromorphic parameters and obtain an effective STS mean residence time. The effective STS mean residence time can then be deconvolved from the transient storage residence time distribution (measured from a tracer test) to obtain an estimate of HTS mean residence time.

\section{Introduction}

Transient storage is the short-term storage of fluid due to the exchange of solutes and suspended particulates in the main flow with (1) recirculating in-stream flow structures, referred to as surface transient storage (STS); and/or (2) the hyporheic zone, referred to as hyporheic transient storage (HTS) (Bencala and Walters, 1983; Boulton et al., 1998; Briggs et al., 2009). By definition, the total transient storage in a stream is the sum of STS and HTS. Transient storage has been known to provide refugia for aquatic communities when poor water quality, predation, strong currents, extreme surface water temperatures, droughts, and floods cause invertebrates to seek shelter in slower moving flows in the surface stream or in interstitial pore spaces of the hyporheic zone (Boulton, 1993; Lancaster and Hildrew, 1993; Brunke and Gonser, 1997). Transient storage also can improve water quality by 
removing metals (e.g., Bencala et al., 1984; Benner et al., 1995; Bencala, 2011); nutrients (e.g., Newbold et al., 1983; De Angelis et al., 1995; Thomas et al., 2001); organic contaminants (e.g., Squillace et al., 1993); and radioactive nuclides (e.g., Cerling et al., 1990). Improved water quality occurs because slower moving water in transient storage zones increases solute residence times (compared to the main channel), increasing the interaction of nutrient-rich surface waters with biogeochemically-reactive sediments (Harvey and Wagner, 2000; McClain et al., 2003; Gooseff et al., 2007).

Numerous tracer studies have strived to develop relationships between the transient storage mean residence time and volume, and various stream geomorphic and hydraulic parameters. The purpose of field-based tracer studies is to gain a better understanding of stream solute transport behavior by accounting for transient storage zones. Previous transient storage studies have focused on relating transient storage to the following hydromorphic parameters: channel order and confinement (D'Angelo et al., 1993; Gabriel and Boufadel, 2002); streambed and aquifer lithology (substrate) (Valett et al., 1996, 1997; Morrice et al., 1997; Argerich et al., 2011); discharge (Hall et al., 2002; Harvey et al., 2003); transient storage area (Mulholland et al., 1997; Laenen and Bencala, 2001; Gücker and Boëchat, 2004; Ensign and Doyle, 2005); channel bed form (Harvey and Bencala, 1993; Gooseff et al., 2003; Anderson et al., 2005; Wörman et al., 2007; Cardenas et al., 2008); wood debris, leaf packs, and vegetation (Gabriel and Boufadel, 2002; Lautz and Siegel, 2006; Lautz et al., 2006; Wondzell et al., 2009b); streambed friction factor (Harvey et al., 2003; Salehin et al., 2003; Zarnetske et al., 2007); stream power per unit width (Zarnetske et al., 2007); channel tortuosity (Kasahara and Wondzell, 2003; Baker et al., 2012); and hyporheic exchange (as determined by hydraulic gradients and streambed-aquifer hydraulic conductivity) (Harvey et al., 1996; Battin et al., 2003; Lautz and Siegel, 2006; Wondzell et al., 2009a,b).

Tracer studies typically use 1-D transient storage models (e.g., OTIS; Bencala and Walters, 1983; Runkel, 1998) to quantify transient storage effects on solute transport because they have the advantage of providing reach-averaged parameter estimates. However, there are a number of drawbacks when using the inverse transient storage modeling approach. First, model parameter estimates are empirical and, therefore, not transferrable to either the same stream under different flow conditions or different stream types (i.e., small versus large streams, headwater, mid-order, or valley streams) (Harvey et al., 2003; Salehin et al., 2003; Stonedahl et al., 2012). Second, transient storage models assume that any mass entering a storage zone will return to the main channel at the location of entrainment, thereby neglecting mass transfer through relatively long hyporheic flow paths (Bencala et al., 2011). Third, reach-averaging a stream's total transient storage parameters couples the effects of STS and HTS, and does not provide information on the relative influence of surface and hyporheic exchange on solute entrainment and retention (Choi et al., 2000; Briggs et al., 2009). Lastly, precise relationships between transient storage, solute exchange, and stream hydromorphic parameters have not been identified as different studies produce contrasting results. The weak relationships observed between transient storage and solute retention may be due to either the complexities associated with transient storage zones (Haggerty et al., 2009), or the inability of current tracer techniques to adequately identify relationships between stream hydromorphic parameters and nutrient uptake and retention when the effects of STS and HTS are coupled (Briggs et al., 2009).

Within the last decade, a number of field and numerical studies have attempted to resolve the issues associated with the inverse modeling approach by quantitatively separating STS from HTS; however, there are drawbacks to each approach. In-stream transport of tracers has increasingly been supplemented with measurements of tracer transport into hyporheic and surface water storage zones (Harvey and Fuller, 1998; Harvey et al., 2005). The goal is to help isolate characteristics of STS and HTS environments, although the comparisons are often limited by the small number of pointscale observations, which may not be representative of storage processes in the reach as a whole. Gooseff et al. (2005) performed tracer tests in two adjacent stream reaches that had comparable physical characteristics with the exception of streambed material: one was underlain by impervious bedrock and the other by a thick alluvium (hyporheic zone). While the longer mean residence time of the alluvial reach clearly showed the impact of HTS on the stream residence time distribution (RTD), a reference stream with insignificant HTS is needed in this approach. Tracer tests by Ensign and Doyle (2005) prior to and after wood debris removal clearly showed a decrease in STS and the impact of STS on the RTD. Nonetheless, the drawbacks to this approach include (1) removing wood debris is labor-intensive; (2) wood debris provides refugia and contains microbial biomass, and their removal can adversely affect the stream ecology; and (3) the streambed was raked, which can change streambed hydraulic properties and disrupt microbial communities on streambed sediments. Gooseff et al. (2008) measured RTDs in the main channel and in twelve STS zones, but did not deconvolve the STS RTDs, resulting in a characteristic power-law tailing behavior. Gooseff et al. (2011) measured and deconvolved STS RTDs from the main channel RTD adjacent to each STS. This method is promising, but can be labor intensive if a large number of STS zones exist. Furthermore, a larger data requirement is needed than was used in the study (e.g., multiple sensors should be placed within a single STS zone) because STS zones are not well mixed and sensors placed in poorly-mixing regions overestimate mean residence time (Jackson et al., 2012). Stofleth et al. (2008) estimated HTS from Darcy's law, which is not constrained by mass balance and can be corrected using the continuity equation. Kasahara and Wondzell (2003), Lautz and Siegel (2006), Gooseff et al. (2006), and Wondzell et 
al. (2009a,b) solved the groundwater flow equation to estimate HTS, and Anderson and Phanikumar (2011) used a 3-D hydrodynamic and particle transport model to generate synthetic STS breakthrough curves (BTCs). O'Connor et al. (2010) estimated STS from predictive equations based on the geometry of emergent lateral cavities at channel sides. The results were used to parameterize a transient storage model using simple a priori physical measurements. A drawback of such approaches is that rigorous data collection is required to obtain enough measurements of streambed topography, hydraulic conductivity and boundary conditions (Gooseff et al., 2006; Wondzell et al., 2009a). Briggs et al. (2009) and Harvey et al. (2005) utilized the two-zone transient storage model - developed by Choi et al. (2000) - to differentiate STS from HTS by measuring tracer breakthrough in the STS and then, utilizing a transient storage model, partially parameterized with STS to determine HTS by inverse modeling. Drawbacks to this approach include (1) additional data collection (e.g., velocity and concentration time series), and (2) the parameterization of two additional parameters, transient storage area and the mass exchange coefficient.

We propose a new approach to quantitatively separate STS from HTS that involves the systematic study of different types of STS from a fluid mechanics perspective. A systematic fluid mechanics approach is proposed because the interplay of fluid dynamics and biogeochemical processes in STS zones influences nutrient uptake, retention, and cycling in stream ecosystems (Lautz and Siegel, 2007; Nepf et al., 2007). Typically, the potential for certain biogeochemical transformations to occur is determined by the RTD (Stanford and Ward, 1988; Boulton et al., 1998). In STS, the RTD that arises depends on the fluid dynamics of mass and momentum exchange (i.e., circulation within the retention region and turbulence level), which influences the mixing and distribution of nutrients and larvae (Jouon et al., 2006; Gooseff et al., 2011).

The fluid mechanics approach will be applied to the study of different types of STS to gain insight into mechanisms driving mass and momentum exchange between the main channel and STS zones. The purpose of the fluid mechanics approach is not to incorporate all of the complexities associated with the flow structure of each STS type, but to identify key hydromorphic parameters (in the mean flow structure) influencing solute mean residence time. In this way, key hydromorphic parameters can be used to develop predictive mean residence time relationships for each type of STS. Predictive relationships may need to be developed for ranges of STS geometries (e.g., width to length aspect ratios) and flow conditions (e.g., Reynolds numbers) because the mean flow field does not significantly change within specified ranges.

The long-term goal is to quantitatively separate STS from HTS by developing an effective STS mean residence time that is based on predictive relationships between fieldmeasurable stream parameters and the mean residence times of different types of STS. The effective STS mean residence time can then be deconvolved from the total transient storage RTD (measured from a tracer test) to obtain an estimate of the HTS mean residence time. As a starting point, a classification scheme is needed to characterize different types of STS in riverine systems based on their flow structure.

The purpose of this paper is to introduce a classification scheme that categorizes different types of STS in riverine systems based on their flow structure. From a fluid mechanics perspective, all STS have flow fields characterized by the formation of a recirculation region comprised of one or more entrained gyres as well as by the formation of at least one free-shear flow: a jet, wake, or mixing layer. Eight types of STS are identified and, in some cases, subcategorized on the basis of differing characteristic mean flow structure: (1) lateral cavities (emergent and submerged); (2) protruding in-channel flow obstructions (backward- and forward-facing step); (3) isolated in-channel flow obstructions (emergent and submerged); (4) cascades and riffles; (5) aquatic vegetation (emergent and submerged); (6) pools (vertically submerged cavity, closed cavity, and recirculating reservoir); (7) meander bends; and (8) confluence of streams (Table 1).

The classification scheme presented is based on review of transient storage literature as well as field observations of rivers and streams. The mean turbulent flow structure characteristic to each STS type is described to provide a basic understanding of the key physical processes influencing exchange dynamics and mean residence time. This paper is not intended to provide a comprehensive review of all fluid mechanics literature. Instead, the classification scheme described is a compilation of previous studies and is meant as a basis for future work and research directions to accurately quantify the effects of STS on stream solute transport.

\section{Background: free shear flows}

Free shear flows are comprised of coherent structures - turbulent features in the flow field of different shapes (i.e., rollers, ribs, horseshoes, and tubes) that can be recognized within the more disordered flow (Socolofsky and Jirka, 2005; Versteeg and Malalasekera, 2007). These coherent structures (larger scale turbulence) are formed in the velocity shear region by instabilities (e.g., Kelvin-Helmholtz, Corcos-Lin, Widnall), where smaller vortices interact by mechanisms such as pairing, tearing, or stretching to form larger scale vortical structures (Socolofsky and Jirka, 2005). KelvinHelmholtz instabilities cause the pairing and growth of vortices as they are advected downstream. Instabilities in the flow structure constantly form and destroy vortices, inducing unsteadiness. However, much information can be obtained by considering the mechanisms of mass and momentum exchange from the mean flow field.

There are three types of free shear flows: jets, wakes, and mixing layers (Versteeg and Malalasekera, 2007). Some STS have flow fields that are a complex interaction between either 
Table 1. Summary of STS classification scheme.

\begin{tabular}{|c|c|}
\hline STS type & Distinguishing characteristics \\
\hline \multicolumn{2}{|l|}{ Lateral cavity } \\
\hline Emergent & Mixing layer spans entrance and recirculation region forms in cavity. \\
\hline Submerged & Overtopping flow parallels main channel flow. \\
\hline \multicolumn{2}{|l|}{ Protruding flow obstructions } \\
\hline Backward-facing step & Recirculation region forms behind obstruction protruding from bank. \\
\hline Forward-facing step & $\begin{array}{l}\text { Recirculation region forms in front of and behind obstruction protruding } \\
\text { from bank. }\end{array}$ \\
\hline \multicolumn{2}{|l|}{ Isolated flow obstructions } \\
\hline Emergent & $\begin{array}{l}\text { Horseshoe vortex in front of and von Kármán vortex street behind } \\
\text { obstruction in flow. }\end{array}$ \\
\hline Submerged & Closed recirculation region behind obstruction in flow. \\
\hline Cascades and riffles & Coalescence of (circular cylinder-type) wake fields. \\
\hline \multicolumn{2}{|l|}{ Aquatic vegetation } \\
\hline Emergent & Coalescence of wake fields and maximum canopy velocity near bed. \\
\hline Submerged & Mixing layer at top of canopy and a monami for flexible canopies. \\
\hline \multicolumn{2}{|l|}{ Pools } \\
\hline Vertically submerged cavity & Pool bathymetry sufficiently below upstream reach bathymetry. \\
\hline Closed lateral cavity & Lateral cavity flow field has a backward- and forward-facing step. \\
\hline Recirculating reservoir & $\begin{array}{l}\text { Jet-like flow: flow enters and generates regions of recirculating flow. } \\
\text { Flow impingement: flow impinges, deflects, and recirculates. } \\
\text { Scour pool: vertical flow enters, scours pool head, and recirculates. }\end{array}$ \\
\hline Meander bends & $\begin{array}{l}\text { Inner bank mixing layer forms recirculation region and point bar and } \\
\text { outer bank mixing layer scours and erodes streambank. }\end{array}$ \\
\hline Confluence of streams & Velocity ratio dictates mixing interface position, mode, and coherence. \\
\hline
\end{tabular}

differing free shear flows or the coalescence of similar free shear flows. Most STS types have a flow structure that is analogous to a type of flow studied in fluid mechanics. The mean flow structure of common wake and mixing layer-type flows, which are studied in fluid mechanics and commonly observed in STS, is described below.

\subsection{Wakes}

In a single wake field generated by a circular cylinder, a horseshoe vortex forms in front of the cylinder and is subjected to large-scale sweeping motions toward and away from the cylinder that alternately shed vortex tubes from each side (Fig. 1, Devenport and Simpson, 1990; Hinterberger et al., 2007). The vortex tubes are advected downstream within mixing layers and roll up behind the cylinder body, forming a von Kármán vortex street comprised of streamwise-oriented rollers (finger vortices) of alternating vorticity signs, i.e., the rollers rotate in opposite directions when shed from each side of the cylinder body (Fig. 1a; Braza et al., 1990; Rai and Moin, 1993). Interactions between rollers in the mixing layers cause Kelvin-Helmholtz instabilities. The advected rollers in the von Kármán vortex street entrain and transport sediment (Kirkil et al., 2006, 2008). Fluid and sediment entrainment also occurs in a recirculation region of lower velocities that forms behind the body (Shen and Diplas, 2008). Sediment scouring and entrainment occur in front of the cylinder because large-scale sweeping motions amplify the turbulence and form a scour hole (Fig. 1b).

The wake field generated by a flat plate (width normal to flow $\gg$ length parallel to flow) has a flow structure similar to a circular cylinder. Only the strength and size of the vortical structures differ. The strength of horseshoe vortex circulation and large-scale sweeping motions in front of a flat plate as well as coherence of alternately shed vortex tubes in the mixing layers are higher compared to a circular cylinder (Kirkil and Constantinescu, 2009). This results in a wider von Kármán vortex street behind the flat plate with larger advected rollers that travel farther downstream and have greater ability to entrain sediments. The rate of scouring in front of the flat plate is faster and the size of the recirculation region behind the flat plate is larger, which also result in a greater ability to entrain solutes and sediments. 
A)

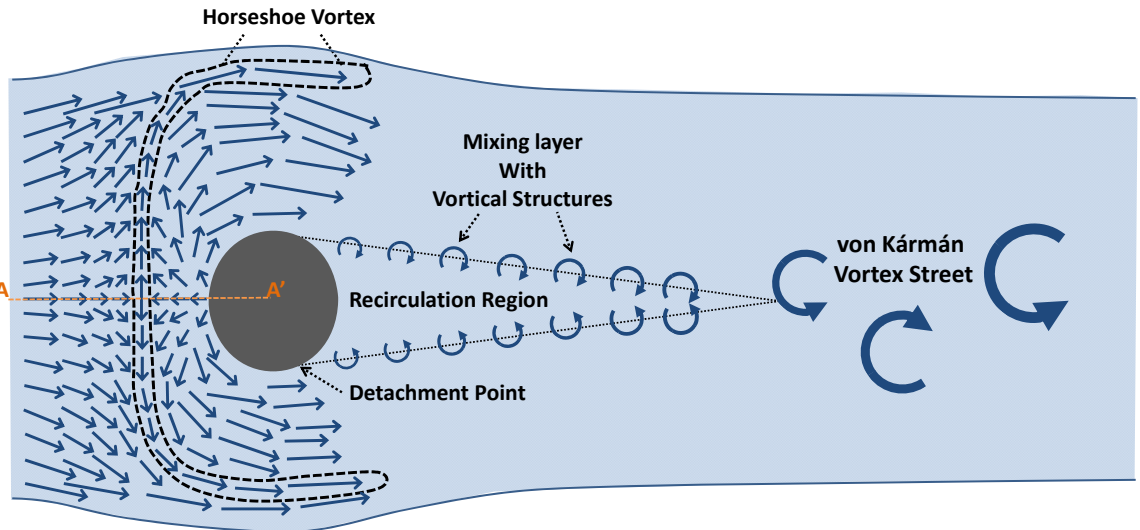

B) $\mathrm{A}$

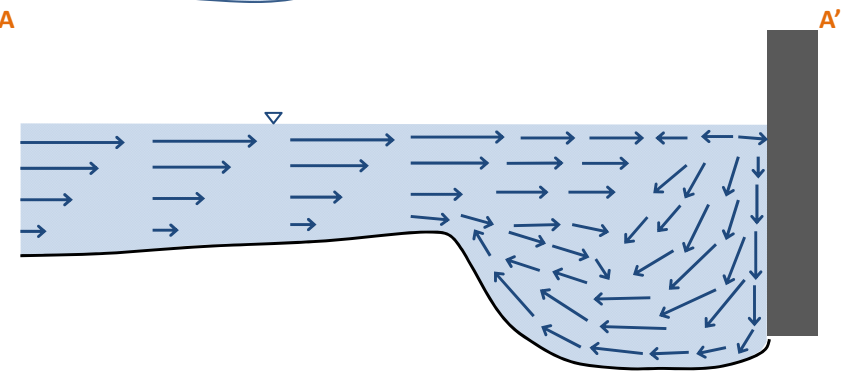

Fig. 1. Plan view (A) and cross-sectional view (B) schematic of flow field around a circular cylinder. (Figure adapted from the work of Kirkil et al., 2006, Hinterberger et al., 2007, and Shen and Diplas, 2008).

\subsection{Recirculation regions enclosed by mixing layers}

Cavity flows (e.g., emergent lateral and vertically submerged cavities) and steps (e.g., backward-facing and forwardfacing) have similar flow structure. For brevity, their generalized flow structure is described herein. Other additional complexities inherent to each of these flows are described in their respective subsections in Sect. 3.

Emergent cavities and steps have flow fields with three characteristic features: (1) shearing and flow separation at their leading edge (upstream corner or detachment point) (Rockwell, 1983; Constantinescu et al., 2009); (2) a mixing layer that forms at the detachment point and impinges at a point downstream (reattachment point); and (3) a recirculation region between the streambank and mixing layer comprised of one or more counter-rotating gyres (see Fig. 2 for visualization) (Rockwell and Naudascher, 1978; Rockwell and Knisely, 1980). Note that the detachment point refers to the location of an adverse (positive) pressure gradient in the main flow direction, which causes the upstream boundary layer to detach from a solid surface (Tritton, 1988). At the detachment point, vortical structures are advected downstream within the mixing layer (Rockwell, 1977, 1998; Lin and Rockwell, 2001). The advected vortices coalesce and grow downstream due to Kelvin-Helmholtz instabilities before impinging on a surface (at the reattachment point), causing the unsteady transport of vorticity into the recirculation region (Sarohia, 1977; Rockwell and Knisely, 1979; Chang et al., 2006). Entrained vortices travel from the downstream to upstream expanse of the recirculation region in a jet-like flow, causing the flow to recirculate (Rockwell, 1998; Lin and Rockwell, 2001).

\section{Classification scheme for surface transient storage}

Eight types of STS were identified in riverine systems and, in some cases, subcategorized on the basis of differing characteristic mean flow structure. The STS classification scheme divides STS into the following categories: (1) lateral cavities (emergent and submerged); (2) protruding inchannel flow obstructions (backward- and forward-facing step); (3) isolated in-channel flow obstructions (emergent and submerged); (4) cascades and riffles; (5) aquatic vegetation (emergent and submerged); (6) pools (vertically submerged cavity, closed cavity, and recirculating reservoir); (7) meander bends; and (8) confluence of streams (Table 1). The mean flow structure characteristic to each STS is described. Current predictive relationships between STS mean residence times and stream hydraulic and morphologic features are presented (if known). For STS types that currently do not have predictive relationships, qualitative relationships are described for key parameters influencing mean residence time and areas are highlighted where further research is needed. 


\section{Plan View}

A) $\quad W / L<0.5$

B) $0.5<W / L<1.5$

C) $\quad W / L>1.5$
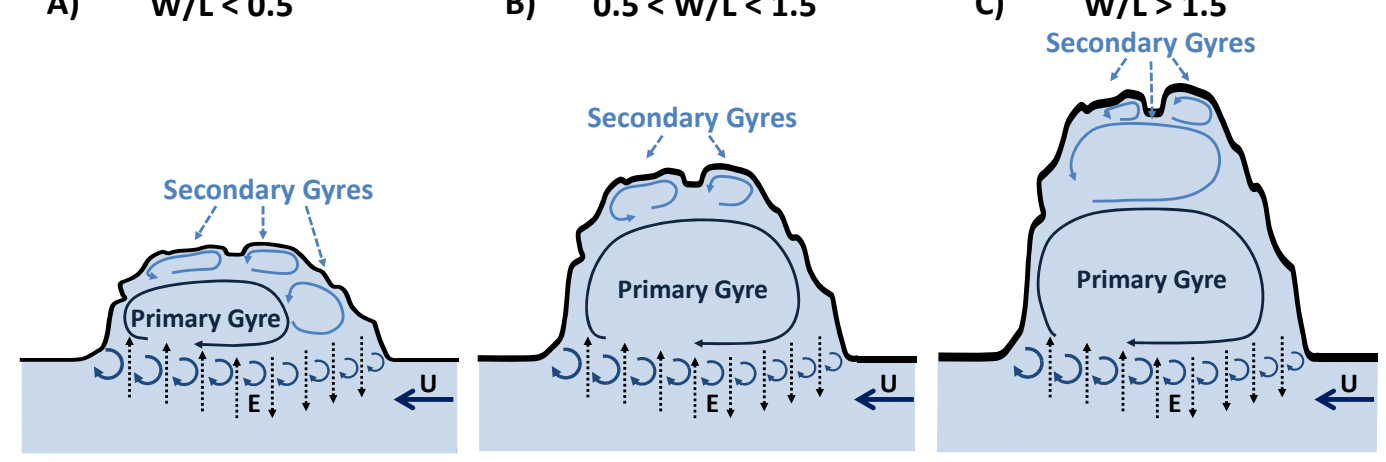

D)

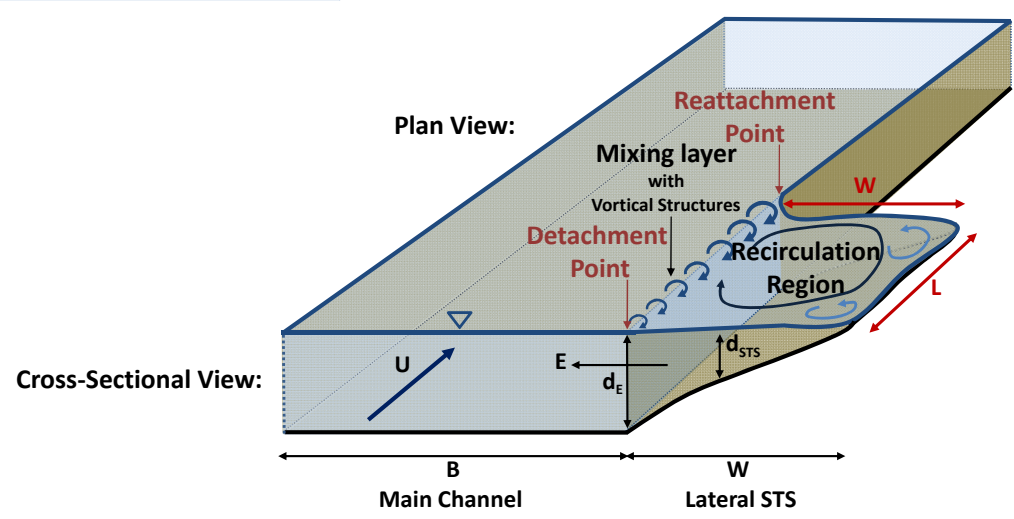

Fig. 2. Plan view schematics of the flow field for an emergent lateral cavity at three $W / L$ aspect ratios and cross-sectional view schematic showing definition of hydromorphic parameters.

\subsection{Lateral cavities}

In rivers and streams, lateral cavities form behind or in front of erosion-resistant obstructions, such as tree roots, logs, and other obstacles that protrude into the flow from the streambank. Lateral cavities also can form as cutouts within bedrock due to either differences in lithology at bedrock contacts, or scouring and erosion along preferential planes of weakness, such as faults or bedding planes. Sequences of man-made lateral cavities, termed groyne fields, also can be found in rivers and are separated by groynes. Groynes are engineered structures comprised of either gravel, stone, earth, or piles and built at an angle to river banks to prevent bank erosion, encourage channel scouring for ship navigation, and enhance sediment storage for fish and vegetation biodiversity (Uijttewaal et al., 2001; Uijttewaal, 2005; Engelhardt et al., 2004; McCoy et al., 2007; Weitbrecht et al., 2008; Yossef and De Vriend, 2011). The flow features associated with lateral cavities are complex and vary, depending on whether the flow obstruction creating the lateral cavity is emergent or submerged.

\subsubsection{Emergent lateral cavities}

Regardless of whether a single or multiple emergent lateral cavities form along a streambank, the flow field is always characterized by flow separation at the leading cavity edge, a mixing layer that spans the entire cavity entrance, and a recirculation region inside the cavity comprised of one or more counter-rotating gyres (Fig. 2; Rockwell and Naudascher, 1978; Rockwell and Knisely, 1980). The recirculation region is comprised of a large primary gyre and may contain one or more smaller, counter-rotating secondary gyres. Secondary gyre formation depends on the width (normal to flow, $W$ ) to length (parallel to flow, $L$ ) aspect ratio of the cavity and irregularities in the wetted perimeter of the cavity (Jackson et al., 2012). As a general rule for rectangular cavities, cavity aspect ratios configure as follows (a) $W / L<0.5$ result in the development of a two-gyre recirculation pattern with the secondary momentum-driven gyre forming in the upstream corner (Fig. 2a); (b) $0.5<W / L<1.5$ result in the development of a one-gyre recirculation pattern (Fig. 2b); and (c) $W / L>1.5$ result in the development of a two-gyre recirculation pattern with the secondary gyre forming adjacent to the primary gyre far from the mixing layer (Fig. 2c; Burggraf, 1966; Ghia et al., 1982; Weitbrecht and Jirka, 2001a; Cheng and Hung, 2006). Note that additional secondary gyres also 
can form due to irregularities in the wetted perimeter of a lateral STS (Jackson et al., 2012).

Within the last decade, many studies have investigated the mean residence time of solutes in lateral STS. A field study by Jackson et al. (2012) investigated the influence of gyre dynamics on mean residence time and found that the mean residence time of a lateral cavity is given by the primary gyre mean residence time and that secondary gyre volume, not mean residence time, contributes to the primary gyre's mean residence time. Langmuir (1908) derived an equation for the mean residence time of a continuous stirred tank reactor, which is analogous to a lateral STS zone (Danckwerts, 1953; Sardin et al., 1991). Uijttewaal et al. (2001) developed a predictive mean residence time for lateral storage zones based on field-measurable parameters by substituting the entrainment hypothesis postulated by Valentine and Wood (1977) into the mean hydraulic residence time formulated by Langmuir (1908):

$\tau=\frac{W L d_{\mathrm{STS}}}{E L d_{\mathrm{E}}}=\frac{W L d_{\mathrm{STS}}}{k U L d_{\mathrm{E}}}$,

where $d_{\mathrm{STS}}$ is the mean depth of the cavity, [L]; and $d_{\mathrm{E}}$ is the mean depth at the mixing layer interface, $[\mathrm{L}] ; U$ is the mean main channel velocity, [L T $\left.{ }^{-1}\right] ; E$ is the exchange velocity leaving the cavity through the mixing layer interface, $\left[\mathrm{LT}^{-1}\right] ; k$ is a dimensionless entrainment coefficient; and $\tau$ is the mean residence time, [T] (Fig. 2d). The first quantity to the right of $\tau$ is the mean residence time derived by Langmuir (1908), the second quantity to the right of $\tau$ is the predictive mean residence time formulated by Uijttewaal et al. (2001), and the entrainment hypothesis assumes that $E=k U$. The entrainment coefficient has a range of variability (between 0.01 and 0.04 ) based on field and experimental studies (Valentine and Wood, 1977; Seo and Maxwell, 1992; Wallast et al., 1999; Uijttewaal et al., 2001; Kurzke et al., 2002; Weitbrecht and Jirka, 2001b; McCoy et al., 2006; Hinterberger et al., 2007; Chang et al., 2007; Weitbrecht et al., 2008; Constantinescu et al., 2009; Jackson et al., 2012). The range of variability measured for the entrainment coefficient is due to the exclusion of parameters, such as streambed roughness, lateral cavity shape, and vegetative drag (Jackson et al., 2012). Recently, Jackson et al. (2013) derived a mean residence time relationship for lateral cavities that incorporates streambed roughness and cavity shape. The relationship uses field-measurable parameters and is applicable to both small streams and larger rivers:

$\tau=21\left[\frac{L^{0.22} W^{0.59} d_{\mathrm{STS}}^{0.59}}{u_{*}^{0.21} U^{0.17} d_{\mathrm{E}}^{0.76} g^{0.25} v^{0.13}}\right]-6.7 \frac{L}{U}$,

where $\tau$ is the mean residence time, $[\mathrm{T}] ; u_{*}$ is the shear velocity, $\left[\mathrm{L} \mathrm{T}^{-1}\right] ; g$ is the gravitational acceleration, $\left[\mathrm{L} \mathrm{T}^{-2}\right]$; and $v$ is the kinematic viscosity, $\left[\mathrm{L}^{2} \mathrm{~T}^{-1}\right]$. This relationship was compared to over sixty field sites measured in six different studies and was found to have a strong linear correlation with an $R^{2}=0.83$ for conservative solutes. However, future work is still needed for non-conservative solute transport.

\subsubsection{Submerged lateral cavities}

Submerged lateral cavities have flow fields similar to emergent lateral cavities with the exception of an additional flow complexity: the upstream flow separates and part of the flow overtops the erosion-resistant obstacle, such as a pair of logs (Fig. 3). Mass and momentum are exchanged by strong, fully three-dimensional vortical structures in the lateral mixing layer and at the water surface in the recirculation region due to the overtopping flow (Tominaga et al., 2001; Uijttewaal, 2005; Yossef and De Vriend, 2011). The overtopping flow disrupts the near-surface recirculation pattern that would otherwise occur for an emergent lateral cavity (Uijttewaal, 2005; McCoy et al., 2007). At low relative submergence levels (i.e., when the ratio of main channel depth to the protruding obstruction height is small), the flow field near the water surface nearly parallels the main channel flow during an overtopping event and then returns to an emergent lateral cavity flow field between events (Uijttewaal, 2005). At higher submergence levels, the near-surface cavity flow remains nearly parallel to the main channel flow (Elawady et al., 2000; Uijttewaal, 2005; Yossef and De Vriend, 2011). The middepth and deeper regions of the recirculating flow are largely unaffected because the deeper cavity flow is driven by momentum exchange through the lateral mixing layer (Peng and Kawahara, 1997; Peng et al., 1999; McCoy et al., 2007). The relative strength of mass and momentum exchange is greater for a submerged lateral cavity when compared to an emergent lateral cavity, resulting in a relatively smaller mean residence time (Tominaga et al., 2001; McCoy et al., 2007, 2008). A majority of the solute exits the cavity via the bottom of the lateral mixing layer interface and at the cavity water surface due to flow overtopping the cavity's downstream flow obstacle (McCoy et al., 2007, 2008). No predictive relationships have been formulated to date to account for the influence of submergence on the mean residence time of a lateral cavity. Nonetheless, the mean residence time of a submerged lateral cavity is dependent on the following parameters:

$\tau=f\left(u_{*}, U, d_{\mathrm{E}}, d_{\mathrm{STS}}, v, g, W, L,\left[d_{\mathrm{C}}-d_{\mathrm{STS}}\right]\right)$,

where $d_{\mathrm{C}}$ is the main channel depth, [L]; and $d_{\mathrm{C}}-d_{\mathrm{STS}}$ is the submergence level, [L].

The hydraulic and morphologic parameters of a submerged lateral cavity (with the exception of submergence level) have the same qualitative relations to mean residence time as the hydraulic and morphologic parameters of an emergent lateral cavity (see Table 2). An increase in either the width, $W$, or length, $L$, of a lateral cavity causes the formation of small secondary gyres, which have slower circulation velocities compared to the large primary gyre and increase mean residence time (Weitbrecht and Jirka, 2001a; Jackson et al., 2012). An increase in the mean cavity depth, 
Table 2. Summary of qualitative relations between mean residence time $(\tau)$ and case-specific parameters for each STS type.

\begin{tabular}{|c|c|c|}
\hline \multirow[b]{2}{*}{ STS type } & \multicolumn{2}{|l|}{ Mean residence time } \\
\hline & Varies proportionally & Varies inversely \\
\hline \multicolumn{3}{|l|}{ Lateral cavity } \\
\hline Emergent & $u_{*}, v, W, L, d_{\mathrm{STS}}$ & $U, g, d_{\mathrm{E}}$ \\
\hline Submerged & $u_{*}, v, W, L, d_{\mathrm{STS}}$ & $U, g, d_{\mathrm{E}},\left(d_{\mathrm{C}}-d_{\mathrm{STS}}\right)$ \\
\hline \multicolumn{3}{|l|}{ Protruding flow obstructions } \\
\hline Backward-facing step & $u_{*}, v, W, x_{\mathrm{BFS}}, d_{\mathrm{STS}, \mathrm{BFS}}$ & $U, g, d_{\mathrm{E}, \mathrm{BFS}}, \gamma$ \\
\hline Forward-facing step & $u_{*}, v, W, x_{\mathrm{BFS}}, x_{\mathrm{FFS}}, y_{\mathrm{FFS}}, d_{\mathrm{STS}, \mathrm{FFS}}, d_{\mathrm{STS}, \mathrm{BFS}}$ & $U, g, d_{\mathrm{E}, \mathrm{FFS}}, d_{\mathrm{E}, \mathrm{BFS}}, \gamma$ \\
\hline \multicolumn{3}{|l|}{ Isolated flow obstructions } \\
\hline Emergent & $u_{*}, v, W, d_{\mathrm{STS}}$ & $U, g, d_{\mathrm{E}}, B, \theta$ \\
\hline Submerged & $u_{*}, v, W, d_{\mathrm{STS}}$ & $U, g, d_{\mathrm{E}}, B, \theta,\left(d_{\mathrm{C}}-d_{\mathrm{STS}}\right)$ \\
\hline Cascades and riffles & $u_{*}, v, D_{\mathrm{g}}, H_{\mathrm{g}}, L_{\mathrm{R}}$ & $U, g, d_{\mathrm{C}}, S_{\mathrm{g}},\left(d_{\mathrm{C}}-H_{\mathrm{g}}\right)$ \\
\hline \multicolumn{3}{|l|}{ Aquatic vegetation } \\
\hline Emergent & $C_{\mathrm{D}}, a, d$ & $U_{\mathrm{c}}, g, \Delta S$ \\
\hline Submerged & $C_{\mathrm{D}}, a, d, h$ & $U_{\mathrm{c}}, g, d_{\mathrm{C}}, \delta_{\mathrm{e}}, S, \Delta U$ \\
\hline \multicolumn{3}{|l|}{ Pools } \\
\hline Vertically submerged cavity & $u_{*}, v, W, L, d_{\mathrm{STS}}$ & $U, g, d_{\mathrm{E}}$ \\
\hline Closed lateral cavity & $u_{*}, v, W, L, d_{\mathrm{STS}, \mathrm{FFS}}, d_{\mathrm{STS}, \mathrm{BFS}}, x_{\mathrm{FFS}}, x_{\mathrm{BFS}}$ & $U, g, d_{\mathrm{E}, \mathrm{FFS}}, d_{\mathrm{E}, \mathrm{BFS}}$ \\
\hline Recirculating reservoir & $u_{*}, v, W, L, d_{\mathrm{STS}}$ & $U, g, d_{\mathrm{E}}, \theta$ \\
\hline Meander bends & $C_{\mathrm{f}}, v, W, L, d_{\mathrm{STS}}, R, B$ & $U, g, d_{\mathrm{E}}, R_{\min }$ \\
\hline Confluence of streams & $C_{\mathrm{f}}, v, W, L, d_{\mathrm{STS}}, R, B, W_{\mathrm{MI}}, d_{\mathrm{MI}}, \beta, U_{1} / U_{2}$ & $g, d_{\mathrm{E}}, R_{\min }$ \\
\hline
\end{tabular}

\section{Plan View}

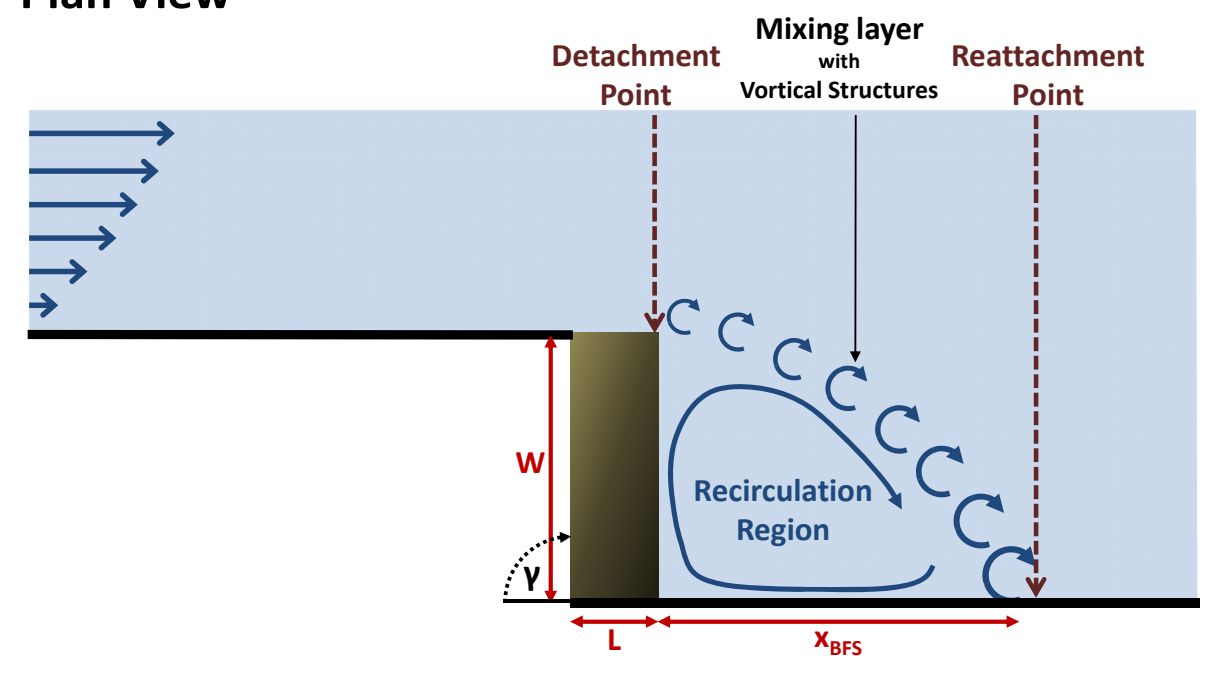

Fig. 3. Schematic of the flow field for a backward-facing step.

$d_{\mathrm{STS}}$, increases the volume for fluid entrainment and mean residence time, whereas an increase in the mean mixing layer depth, $d_{\mathrm{E}}$, increases the mixing layer cross-sectional area for mass and momentum exchange and decreases mean residence time (McCoy et al., 2007, 2008). The main channel velocity and gravitational acceleration drive exchange across the mixing layer interface and decrease mean residence time (Valentine and Wood, 1977). The shear velocity and kinematic viscosity quantify frictional forces that reduce exchange across the mixing layer interface and increase mean residence time (Jackson et al., 2013). 


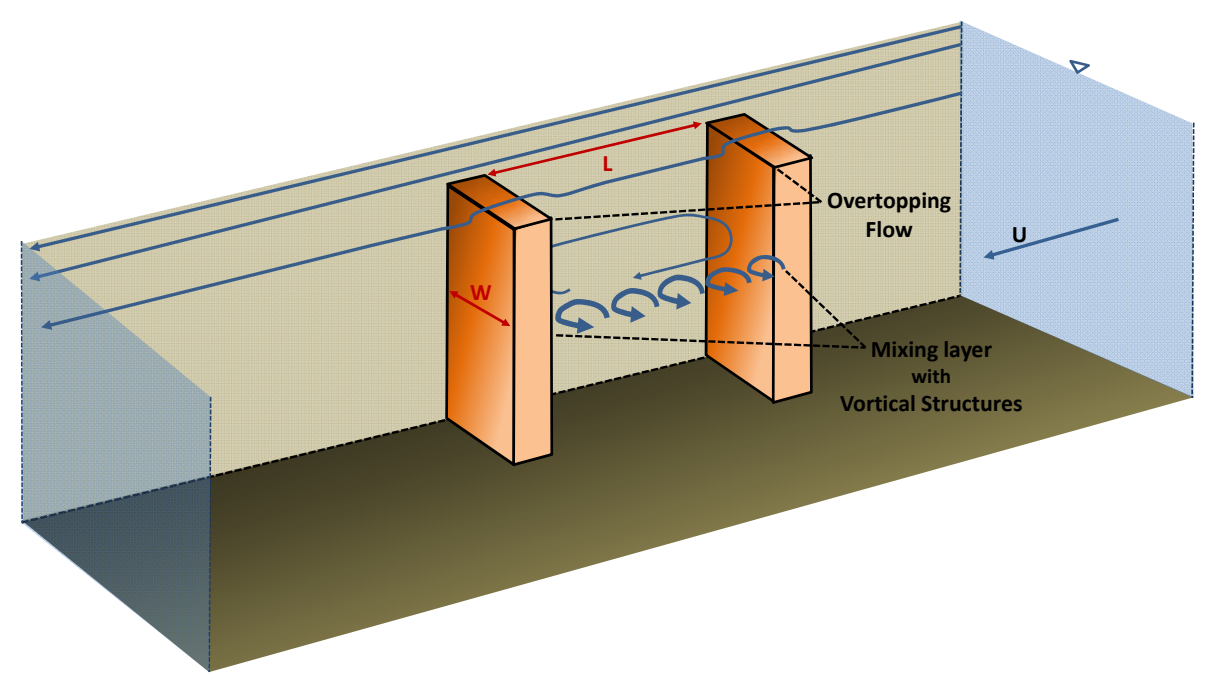

Fig. 4. Schematic of the flow field for a submerged lateral cavity.

\subsection{Protruding in-channel flow obstructions}

Isolated protruding logs or boulders extending from the streambank into the main channel flow generate flow fields equivalent to backward-facing and forward-facing steps in front of and behind the obstacle, respectively. As an example, in situ flow deflection log jams, identified by Abbe and Montgomery (2003) as one type of wood debris in forested streams, form both backward- and forward-facing steps. Backward- and forward-facing steps also can develop in conjunction with an emergent lateral cavity formed by closely spaced obstructions protruding into the main channel (e.g., McCoy et al., 2006). Field studies have found that sediment entrainment within the recirculation regions of backwardand forward-facing steps results in bar development that resembles flood plain deposits (Abbe and Montgomery, 2003). The flow structure of emergent backward- and forwardfacing steps are described below. We note that submerged backward- and forward-facing steps also can occur, especially at high flows. In addition, underflow can occur beneath either a floating $\log$ or a $\log$ situated atop an uneven streambed. However, no work has been done to date to determine the influence of either submergence or underflow on the flow structure of a backward- or forward-facing step, and these cases are not discussed.

\subsubsection{Backward-facing step}

The backward-facing step is characterized by flow separation at the obstacle head, a reattachment point at the streambank that returns the open channel flow to a fully developed state, and a recirculation region on the downstream side of the obstacle located near the streambank (Fig. 4; Kim et al., 1979; Silveira Neto et al., 1993; Hung et al., 1997; Fessler and Eaton, 1999). The recirculation region is delineated by a zero vorticity boundary and the farthest upstream and downstream locations of zero vorticity correspond to the detachment and reattachment points, respectively (Kim et al., 1979; Williams and Baker, 1997). Gyre formation within the recirculation region is comparable to the lateral cavity at the same $W / L$ with the exception that the mixing layer forms at an oblique angle to the channel flow, which elongates gyres within the downstream region. The dimensionless STS zone length (reattachment distance) is given by $x_{\mathrm{BFS}} / W$, where $x_{\mathrm{BFS}}$ is the distance downstream from the obstruction (backward-facing step) detachment point, [L]; and $W$ is the flow obstruction width protruding into the flow, [L] (Fig. 4). The reattachment distance is highly dependent on Reynolds number and varies proportionally (Armaly et al., 1983; Williams and Baker, 1997). Increasing the Reynolds number moves the reattachment point farther downstream because higher in-channel flow velocities increase shearing across the mixing layer interface, causing higher momentum-driven vortical structures to travel farther downstream prior to impingement. The reattachment distance also varies proportionally to the channel expansion ratio with $x_{\mathrm{BFS}} / W$ asymptotically approaching smaller values as the channel expansion ratio is decreased (Kuehn, 1980; Durst and Tropea, 1981; Hung et al., 1997). Due to the high dependence of reattachment distance on hydromorphic parameters, the reattachment distance is still determined empirically and must be measured in the field.

The mean residence time of a backward-facing step is largely dependent on the flow obstruction width $(W)$, Reynolds number, and inclination angle, $\gamma$ (angle measured from the upstream streambank to the obstacle; see Fig. 4). An increase in $W$ can increase recirculation region size, depending on the upstream flow conditions. Thus, larger flow obstruction widths have the potential to increase the volume for fluid entrainment and mean residence time. The recirculation region volume also increases as Reynolds number is 
increased; however, higher main channel flow also increases the strength of vortical structures in the mixing layer, thereby increasing mass exchange and decreasing mean residence time. The angle of the protruding in-stream flow obstacle, $\gamma$, influences the size and strength of the recirculation region that forms behind the obstacle. For protruding obstacles angled upstream into the flow, an increase in $\gamma$ increases the shearing strength at the separation point, meaning that vortical structures within the mixing layer have greater coherence (and momentum). This increases momentum transfer into the recirculation region, which increases the circulation of gyres inside the region and decreases mean residence time. The mixing layer also grows farther downstream before impinging on the streambank at higher inclination angles, increasing the size of the recirculation region (Chen et al., 2006), which may either enlarge the primary gyre or induce secondary gyre formation, subsequently reducing mean residence time. Therefore, the effect of inclination angle on mean residence time is poorly understood because mean residence time may increase or decrease, depending on the relative influence of the angle on mixing layer exchange and recirculation region growth. We hypothesize that the inclination angle will have a stronger inverse relationship. A 3-D numerical study by Chen et al. (2006) showed that inclination angles less than $15^{\circ}$ are the limiting case for the formation of a mixing layer and recirculation region behind the obstacle. Mean residence time varies proportionally to the length $\left(x_{\mathrm{BFS}}\right)$ and depth $\left(d_{\mathrm{STS}, \mathrm{BFS}}\right)$ of the backward-facing step and varies inversely to the mean depth at the mixing layer interface $\left(d_{\mathrm{E}, \mathrm{BFS}}\right)$. Thus, the mean residence time of a backward-facing step is dependent on the following parameters (Table 2):

$\tau=f\left(u_{*}, U, d_{\mathrm{E}, \mathrm{BFS}}, d_{\mathrm{STS}, \mathrm{BFS}}, v, g, W, x_{\mathrm{BFS}}, \gamma\right)$,

noting that neither the submergence level, $d_{\mathrm{C}}-d_{\mathrm{STS}}$, nor underflow is accounted for in Eq. (4).

\subsubsection{Forward-facing step}

The flow dynamics associated with turbulent flow past a forward-facing step are more complex than that of a backward-facing step because, unlike a backward-facing step, a forward-facing step has two regions of flow separation: one region upstream of the step (obstacle) and one region at the step corner (head of obstacle protruding into the flow) (Fig. 5). Upstream flow separation occurs when the incoming turbulent boundary layer separates from the channel wall before approaching the obstacle due to an adverse pressure gradient imposed by the obstacle (Farabee and Casarella, 1986; Pearson et al., 2001). The separated boundary layer impinges at a point along the obstacle and forms a recirculation region in the corner between the obstacle and streambank. For a flow obstacle oriented normalto flow of width, $W$ (Fig. 5a), the upstream detachment distance (from the obstacle), $x_{\mathrm{FFS}}$, and reattachment distance (from the streambank), $y_{\mathrm{FFS}}$, are weakly dependent on Reynolds number, $R e_{\mathrm{W}}$, (based on obstacle width) for $4000<R e_{\mathrm{W}}<26300$ (Awasthi, 2012). The upstream detachment distance ranges from about 0.8 to $1.2 \mathrm{~W}$ and the reattachment distance ranges from about 0.5 to $0.6 \mathrm{~W}$ (Addad et al., 2003; Fiorentini et al., 2007; Camussi et al., 2008; Leclercq et al., 2009).

The second separation region at the obstacle head results in a more complex flow field than the upper boundary layer separation region. Reattached flow travels along the flow obstacle and separates at the obstacle head, where a mixing layer forms and sheds vortices downstream (Kiya and Sasaki, 1983). The flow field downstream of the obstacle head is dependent on the orientation of the obstacle's protrusion into the flow. If a protruding obstacle is oriented such that its width protruding into the flow is much longer than its length (parallel to flow) (e.g., a log oriented normal to flow), then flow separation at the obstacle head forms a mixing layer whose downstream impingement forms the flow field of a backward-facing step (Fig. 5a). However, if a protruding obstacle is oriented such that its width protruding into the flow is much shorter than its length (e.g., $\log (\mathrm{s})$ oriented parallel to flow), then flow separation at the obstacle head forms a mixing layer whose impingement at a point on the obstacle's length forms a recirculation region (Fig. 5b; Farabee and Casarella, 1986). One example of this flow type is logs oriented parallel to the channel axis, which typically form in higher gradient, semi-confined stream reaches and have been identified as bench jams (Abbe and Montgomery, 2003).

In the case of an obstacle oriented parallel to flow (Fig. 5b), the downstream reattachment distance of the recirculation region is dependent on $R e_{\mathrm{W}}, W$, and inclination angle, $\gamma$, into the flow. Studies of forward-facing steps typically relate reattachment distance to the flow obstacle geometry using the hydromorphic ratio $W / \delta$, where $\delta$ is the boundary layer displacement thickness. Exact relationships between flow and geometry parameters and downstream attachment distance are poorly understood due to the unsteady nature of the mixing layer at the obstacle head and the amplification of mixing layer instabilities from the upstream recirculating flow and mixing layer (Pearson et al., 2001; Awasthi, 2012). A generalized result for reattachment distance is that $W / \delta=1.43$ yields reattachment distances ranging between 4.7 and $5.0 \mathrm{~W}$ for $17000<R e_{\mathrm{W}}<50000$ (Moss and Baker, 1980; Addad et al., 2003; Gasset et al., 2005), and decreases to $3.2 \mathrm{~W}$ at higher $R e_{\mathrm{W}}(=170000)$ (Leclercq et al., 2009). At lower $\mathrm{W} / \delta(\sim 0.2)$, reattachment distances range between 1.5 and $2.1 \mathrm{~W}$ for $8000<R e_{\mathrm{W}}<26300$ (Camussi et al., 2008), and decrease to $1.4 \mathrm{~W}$ at higher $R e_{\mathrm{W}}(=50000)$ (Castro and Dianat, 1983). Thus, there is a general trend such that increasing obstacle protrusion width into the flow increases reattachment distance and increasing Reynolds number decreases the reattachment distance. 


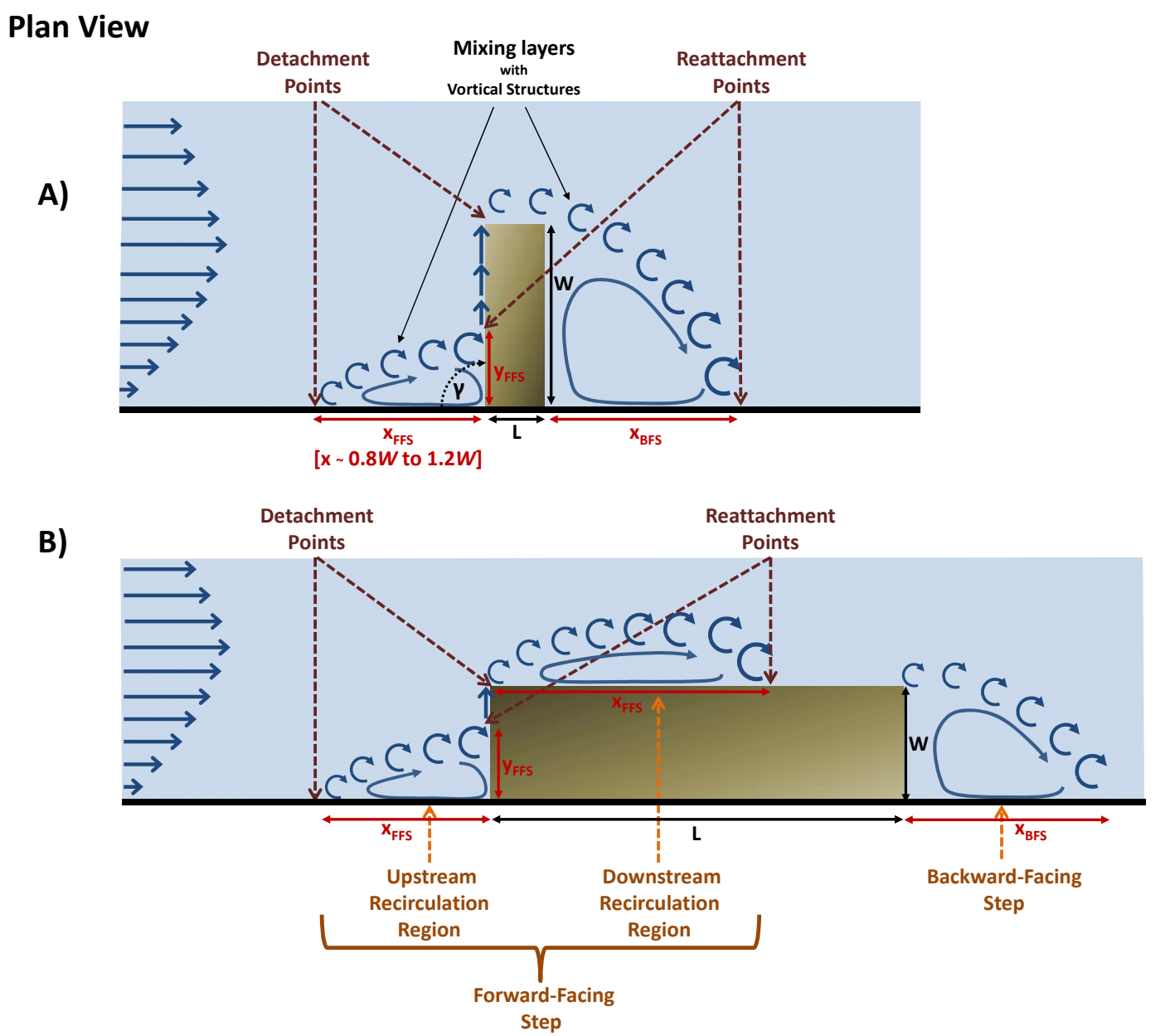

Fig. 5. Schematics of the flow field for a forward-facing step with two different in-stream flow obstruction orientations: (A) $W>L$ and (B) $W<L$.

The mean residence time of recirculation regions upstream and downstream of the obstacle is dependent on a number of parameters. The size of the upstream recirculation region is weakly dependent on Reynolds number; however, the circulation of gyres (and mean residence time) in the recirculation region is driven by shearing in the mixing layer, which is dependent on Reynolds number (i.e., increased mixing decreases residence time). The obstacle inclination angle also can influence recirculation region size and the number of gyres in the region. Increasing the number of gyres increases the mean residence time because smaller, counter-rotating secondary gyres have slower circulation compared to the primary gyre (Jackson et al., 2012). Reynolds number and protruding obstacle width have more effect on the downstream recirculation region. Increasing obstacle protrusion width or decreasing Reynolds number will increase downstream recirculation region size, which increases primary gyre size in the recirculation region and, thus, mean residence time. An increase in free-stream velocity increases $x_{\mathrm{FFS}}$, which increases recirculation region size and mean residence time.
Larger $y_{\mathrm{FFS}}, d_{\mathrm{STS}, \mathrm{FFS}}$, and $d_{\mathrm{STS}, \mathrm{BFS}}$ also increase upstream recirculation region size, which increases the volume for fluid entrainment and mean residence time. Larger $d_{\mathrm{E}, \mathrm{FFS}}$ and $d_{\mathrm{E}, \mathrm{BFS}}$ increase momentum exchange and gyre circulation, which decreases mean residence time. For the downstream recirculation region, the inclination angle will have similar effects for the forward-facing step as in the backward-facing step where, as discussed in the previous section, mean residence time will either increase or decrease, depending on upstream flow conditions. Thus, the exact influence of larger inclination angles on mean residence time is poorly understood. Again, we hypothesize that the inclination angle will have a stronger inverse relationship. In summary, the mean residence time of a forward-facing step is dependent on the following parameters (Table 2):

$\tau=f\left(u_{*}, U, d_{\mathrm{E}, \mathrm{FFS}}, d_{\mathrm{E}, \mathrm{BFS}}, d_{\mathrm{STS}, \mathrm{FFS}}, d_{\mathrm{STS}, \mathrm{BFS}}, v, g, W, x_{\mathrm{BFS}}, x_{\mathrm{FFS}}, y_{\mathrm{FFS}}, \gamma\right)$

Note that the submergence level, $d_{\mathrm{C}}-d_{\mathrm{STS}}$, and underflow are not accounted for in Eq. (5), and that two predictive mean 


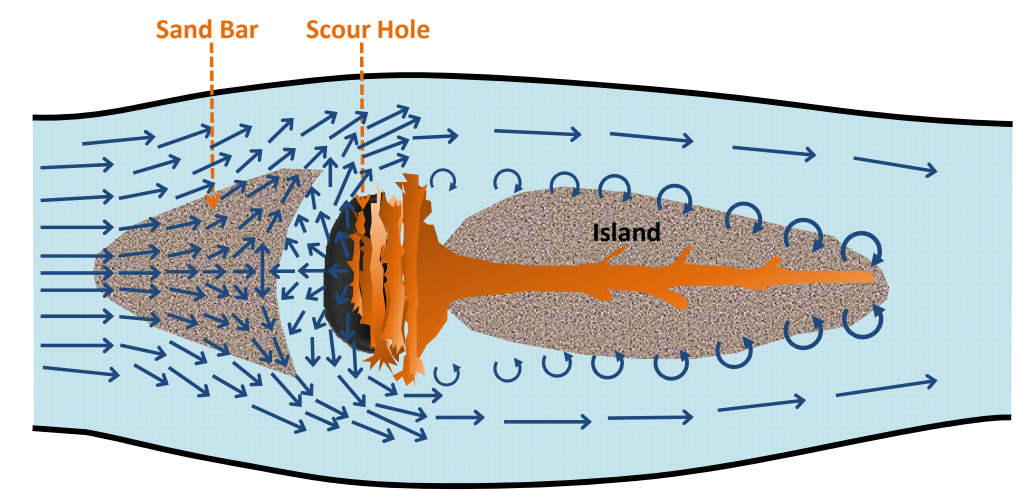

Fig. 6. Bar apex jam: example flow field for an isolated in-channel flow obstruction. (Figure adapted from the work of Abbe and Montgomery, 1996, 2003.)

residence time relationships may be needed to represent the upstream and downstream recirculation regions.

\subsection{Isolated in-channel flow obstructions}

Isolated in-channel flow obstructions are defined as any flow obstacle that is surrounded by main channel flow on all sides, such as a large boulder, log, or an amalgamation of wood debris. This type of flow is analogous to flow past a blunt body, which generates a single wake field. The flow structure is similar for flow obstacles of different shapes. Isolated in-channel flow obstructions can be either emergent or submerged. Note that no work has been done to date to estimate residence times in wake fields behind emergent or submerged blunt bodies and that we provide hypotheses for qualitative relations between different hydraulic and morphologic parameters and mean residence time.

\subsubsection{Emergent isolated in-channel flow obstructions}

Flow in the vicinity of an isolated boulder (Fig. 1) or log is characterized by turbulent flow around a circular cylinder or flat plate, respectively, as described in Sect. 2.1. The recirculation region formed behind isolated boulders and logs entrain solutes and sediments. If the cross-stream length of the flow obstacle is of sufficient size, meaning that the boulder or $\log$ encompasses a large fraction of the channel width, then the recirculation region can form sand bars and islands in front of and behind the obstacle, respectively. For example, a bar apex jam - a type of wood debris identified by Abbe and Montgomery (1996) - is an amalgamation of wood debris oriented normal to flow and centered in the channel. Main channel flow either diverges around the jam or impinges and deflects on the upstream side of the jam in the vertical and transverse directions (Fig. 6). Vertical flow deflection and impingement on the streambed forms a large scour hole on the upstream side of the jam (see Fig. 1b). Large-scale sweeping motions in and out of the scour hole alternately shed vortices downstream of the jam, forming a turbulent wake field. A large recirculation region forms behind the jam and sediment entrainment in this region can form an island. Transverse flow deflection causes fluid to either flow around the jam or to temporarily traverse upstream in a strong backward flow toward the oncoming streamflow. A stagnation point forms upstream of the scour hole and is centered above an arcuate bar where the horseshoe vortex forms.

Solute entrainment and mean residence time of isolated emergent obstacles is dependent on a number of factors. The most influential factor is the size of the obstacle. The relative fraction of the obstacle width (normal to flow), $W$, to the channel width, $B$, determines the size of the recirculation region behind the obstacle and whether an arcuate bar forms upstream beneath the horseshoe vortex. Thus, we hypothesize that an increase in $W$ or decrease in $B$ will cause an increase in mean residence time. Either one or two mean STS depths $\left(d_{\mathrm{STS}}\right)$ can be defined, depending on whether the ratio $W / B$ is sufficient for bar formation upstream of the obstacle. An increase in $d_{\text {STS }}$ will increase the volume of fluid entrainment, which will increase mean residence time. Other variables of importance are the mean mixing layer depth, freestream velocity, and porosity of the emergent obstacle, $\theta$. Mean mixing layer depth $\left(d_{\mathrm{E}}\right)$ and free-stream velocity $(U)$ increase mass and momentum exchange, which decreases mean residence time. Porosity is important in the case of wood debris where flow through the dam can disrupt the coherence of the wake field, which decreases mean residence time. Thus, the mean residence time of isolated emergent obstacles is dependent on the following parameters (Table 2):

$\tau=f\left(u_{*}, U, d_{\mathrm{E}}, d_{\mathrm{STS}}, v, g, W, B, \theta\right)$.

\subsubsection{Submerged isolated in-channel flow obstructions}

Submerged isolated obstacles have the additional complexity of flow overtopping the obstacle and disrupting the wake field (Fig. 7). Upstream of the obstacle, larger submergence depths change the position and size of the horseshoe vortex. The horseshoe vortex moves closer to the obstacle and decreases in size as the submergence depth increases due 


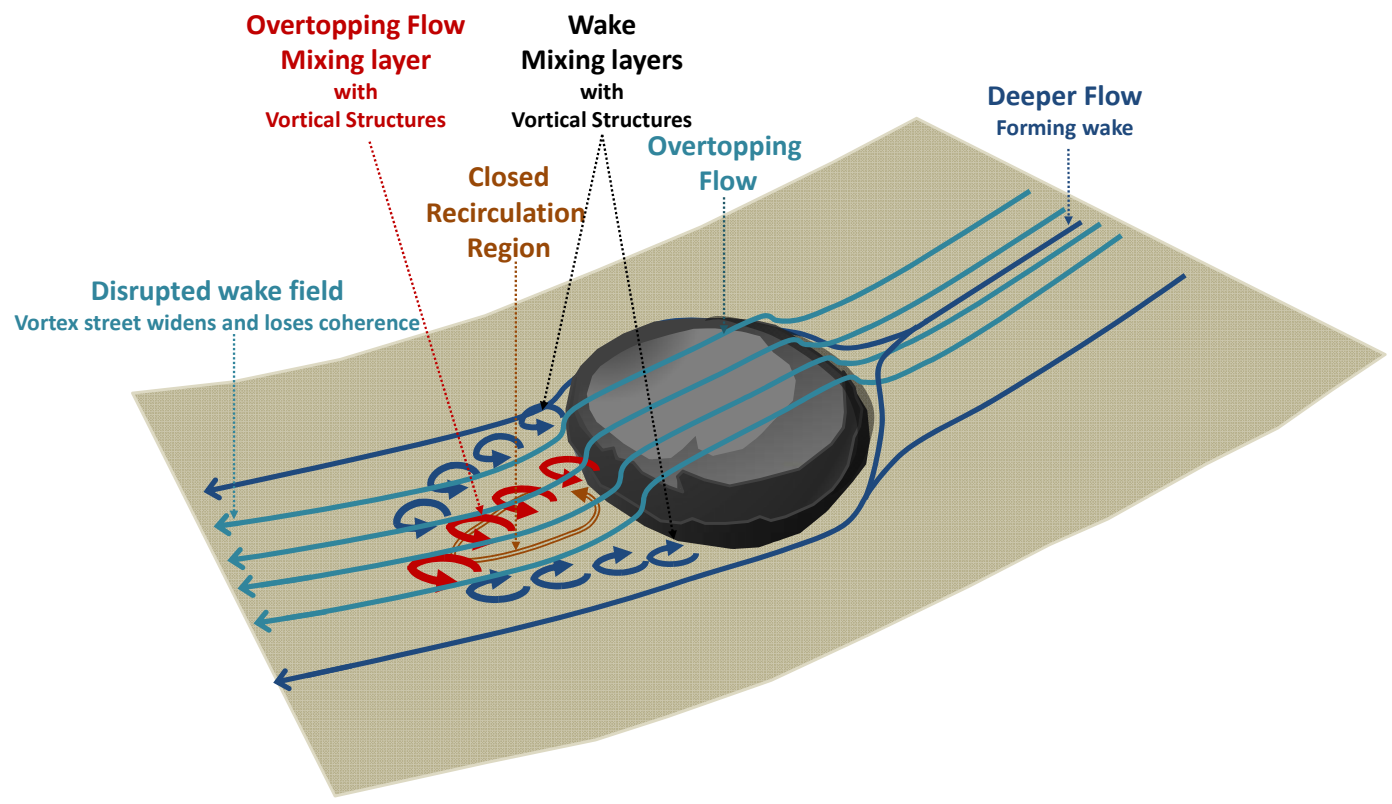

Fig. 7. Schematic of the flow field for a submerged isolated in-channel obstacle.

to a weakening of the backward flow as more flow overtops the obstacle (Sadeque et al., 2008). The flow accelerates as it overtops the obstacle, causing the destruction of wake vortices directly behind the obstacle. However, at higher submergence depths, flow separation occurs at the downstream edge of the obstacle, forming a mixing layer. KelvinHelmholtz instabilities in the mixing layer form large-scale vortical structures that impinge on the streambed and transport vorticity behind the obstacle, forming a closed recirculation region. The length of the recirculation region increases as the flow depth increases once the recirculation region has formed. The width of the vortex street also increases as the flow depth increases, but vortex coherence and circulation weakens (Sadeque et al., 2008).

The spatial distribution of solute entrainment differs between emergent and submerged isolated obstacles. In the emergent case, the potential for solute and sediment entrainment is high because of the relatively high vortex street coherence compared to the submerged case. In the submerged case, the potential for solute and sediment entrainment decreases because vortex street coherence weakens as flow overtops the obstacle. Higher submergence depths cause the flow to be nearly parallel to main channel flow in the nearsurface region behind the obstacle, which decreases the size of the recirculation region and mean residence time. The closed recirculation region behind the obstacle is driven by mixing layer momentum exchange. Lower flow rates decrease exchange and increase mean residence times. All qualitative relations between hydraulic and morphologic parameters and mean residence time for the case of an isolated emergent obstacle hold for the case of submergence. Thus, the mean residence time of isolated submerged obstacles is dependent on the following parameters (Table 2):

$$
\tau=f\left(u_{*}, U, d_{\mathrm{E}}, d_{\mathrm{STS}}, v, g, W, B, \theta, d_{\mathrm{C}}-d_{\mathrm{STS}}\right) .
$$

\subsection{Cascades and riffles}

Cascades and riffles are relatively straight, steep, shallow reaches with higher flow velocities compared to adjacent geomorphic features, such as pools or steps (Montgomery and Buffington, 1997; Raven et al., 1998; Kang and Sotiropoulos, 2011). Cascade and riffle reaches typically are comprised of heterogeneously-spaced roughness elements, such as gravels, cobbles, or boulders, which have relatively large roughness heights - ratio of grain height, $H_{\mathrm{g}}$, to mean water depth, $d_{\mathrm{C}}$. Cascades and riffles are regions of high turbulent mixing and can be characterized by the coalescence of wake fields behind individual circular roughness elements (i.e., circular cylinders). In a coalesced turbulent wake field, the wake field generated by one roughness element interacts with adjacent wake fields from nearby roughness elements. Advected rollers from the mixing layers induce the formation of anisotropic small-scale structures that interact to form complex wake fields (Tritico and Hotchkiss, 2005; Kang and Sotiropoulos, 2011). Closely spaced and/or heterogeneous distributions of roughness elements and bedform features can destabilize individual wake fields, causing the rollers to lose their coherence and ability to entrain solutes (Constantinescu et al., 2013). The rollers also can lose their coherence at higher flow depths due to flow overtopping individual roughness elements; however, smaller closed recirculation regions can form as in the case of submerged flow 
obstructions (discussed above in Sect. 3.3.2). At high discharge, bed armoring is common along cascade and riffle reaches because high turbulent mixing (i.e., high transverse Reynolds shear stresses and turbulent kinetic energies) in the jet-like flow between roughness elements prevents deposition of finer sediment (Vanoni, 1975; Hirsch and Abrahams, 1981; Sear, 1996).

Cascades and riffles have fast exchange rates and relatively small mean residence times behind individual roughness elements (Kang and Sotiropoulos, 2011). However, solute entrainment within the coalesced wake field can contribute to reach-averaged estimates of mean residence time, as observed in a laboratory experiment of four riffle-pool reaches by Seo and Maxwell (1992), where gravel (one particle thick) on the riffle reaches had a small contribution to the flume-length averaged RTD. Thus, mean residence time varies proportionally to cascade/riffle reach length, $\left(L_{\mathrm{R}}\right)$. Furthermore, the contribution of cascade and riffle reaches to reach-averaged mean residence times may be significant, depending on the mean grain diameter $\left(D_{\mathrm{g}}\right)$, spacing between roughness elements $\left(S_{\mathrm{g}}\right)$, channel velocity $(U)$, mean flow depth $\left(d_{\mathrm{C}}\right)$, and the mean submergence depth of the roughness elements $\left(d_{\mathrm{C}}-H_{\mathrm{g}}\right)$. Mean residence time varies proportionally to $D_{\mathrm{g}}$ and $H_{\mathrm{g}}$ because these parameters increase the volume of closed recirculation regions for fluid entrainment. An increase in roughness element spacing reduces wake interactions and turbulence associated with fluid entrainment, which decreases mean residence time. An increase in channel velocity increases momentum exchange and mean residence time, whereas an increase in mean channel depth and submergence depth decrease the size of individual recirculation regions and mean residence time. In summary, the mean residence time of cascade and riffle reaches is dependent on the following parameters (Table 2):

$\tau=f\left(u_{*}, U, d_{\mathrm{C}}, H_{\mathrm{g}}, d_{\mathrm{C}}-H_{\mathrm{g}}, v, g, L_{\mathrm{R}}, D_{\mathrm{g}}, S_{\mathrm{g}}\right)$.

\subsection{Aquatic vegetation}

Submerged and emergent aquatic macrophytes are common in rivers, wetlands, estuaries, marshes, and streams, and have been well studied by Nepf, Ghisalberti, and coworkers. Seagrass meadows, dense algal mats (i.e., kelp forests), and mangroves are all types of aquatic vegetation canopies. Aquatic vegetation promotes sediment deposition, increases solute residence times, and enhances water quality through vertical mixing gradients due to increased drag and reduced shear stress near the streambed of the canopy (Nepf, 1999; Ghisalberti and Nepf, 2002, 2005, 2006; Harvey et al., 2005, 2009). Emergent and submerged aquatic macrophytes have differing physical characteristics and, therefore, differing influences on the main channel flow field. Emergent macrophytes have maximum heights that extend above the water surface and (typically) rounder stem geometries to increase their rigidity and strength against oncoming flow, whereas submerged macrophytes have maximum heights below the

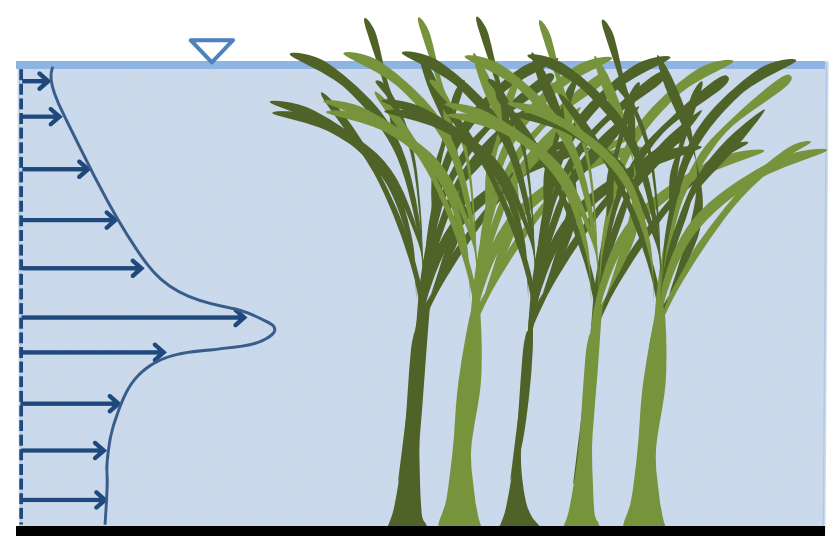

Fig. 8. Schematic of the flow field for emergent aquatic vegetation. (Figure adapted from Nepf, 2012.)

water surface and flatter blade-like stems to increase their elasticity so they can move with the main channel flow (Nepf, 2012). Thus, emergent macrophytes can be considered bluff bodies and submerged macrophytes can be considered streamlined bodies.

\subsubsection{Emergent aquatic vegetation}

The flow field associated with emergent aquatic vegetation is characterized by the coalescence of wake fields behind individual stems (Finnigan, 2000). As emergent canopies fill the entire flow depth, the mean velocity profile and turbulence characteristics of the main channel flow are dependent on the density (spacing) and frontal area geometry of individual macrophytes (Finnigan, 2000; Bennett et al., 2002). The mean velocity profile has a maximum canopy velocity near the streambed due to stem branching (Fig. 8). The turbulent length scale is set by either the mean spacing between individual plant stems $(\Delta S)$ or the stem diameter $(d)$, depending on whichever scale is smaller (Tanino and Nepf, 2008). Turbulence in the stem wake region is higher than turbulence generated in the boundary layer region of the streambed, represented as bed shear stress (Nepf, 1999). Therefore, the turbulent kinetic energy produced, which is dependent on the ratio of stem drag in the wake region to viscous drag, scales with the stem drag (Nepf and Koch, 1999). The turbulence intensity in the wake region varies proportionally with increasing stem density (Gambi et al., 1990).

The flow structure imposed by emergent aquatic vegetation is complex. Flow and mass transport equations have been derived by double-averaging the Navier-Stokes equations (Nikora et al., 2001, 2007; Nepf, 2012). The transport equations describe fluid transport through an emergent aquatic canopy using a diffusion coefficient, $D$, which is the sum of the turbulent diffusion coefficient and mechanical diffusion coefficient, which accounts for tortuosity of flow paths (Nepf, 1999). In sparsely vegetated canopies, turbulent diffusion dominates and $D=0.2 U_{\mathrm{c}} d$, where $U_{\mathrm{c}}$ is 


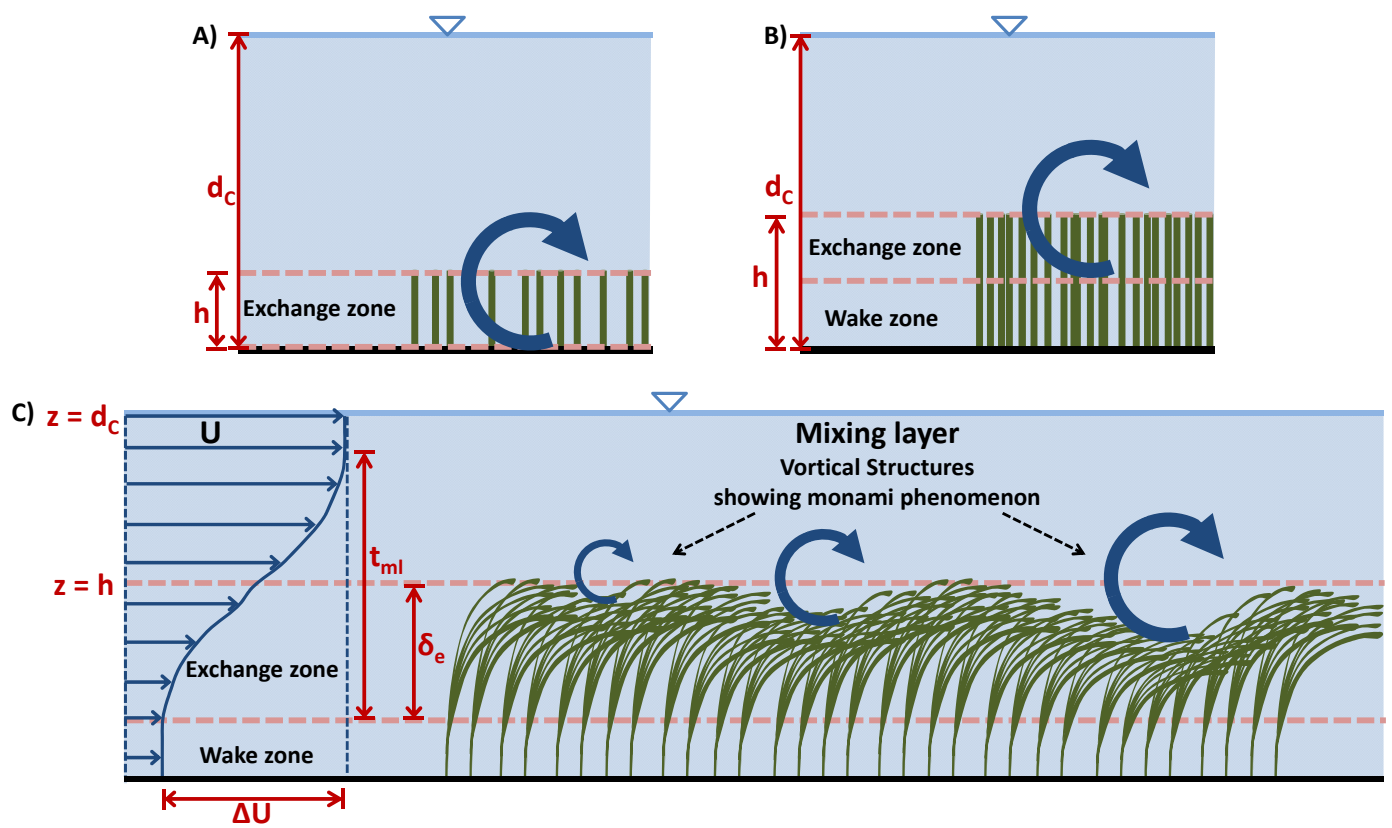

Fig. 9. Schematics of the flow field for submerged aquatic vegetation. (A) Influence of deep submergence and sparse canopy on mixing layer penetration depth. (B) Influence of shallow submergence and a dense canopy on mixing layer penetration depth. (C) Flow structure within a flexible submerged canopy showing monami phenomenon. (Figure adapted from Ghisalberti and Nepf, 2002, 2005, Nepf et al., 2007, Nepf and Ghisalberti, 2008, and Nepf, 2012.)

the mean canopy velocity, $\left[\mathrm{L} \mathrm{T}^{-1}\right]$ (Lightbody and Nepf, 2006). In densely vegetated canopies, the influence of turbulent diffusion decreases and mechanical diffusion becomes the dominate diffusion process, especially at higher Reynolds numbers. The mechanical diffusion coefficient is given by $D / U_{\mathrm{c}} d=a d$, where $a$ is the frontal area per canopy volume, [L $\left.{ }^{-1}\right]$ (Nepf et al., 2007).

Several relationships have been found in the literature relating different hydromorphic parameters. Mean flow velocity $\left(U_{\mathrm{c}}\right)$ varies inversely with the front area (and diameter) of individual stems, vegetation density, and the canopy drag coefficient $\left(C_{\mathrm{D}}\right)$ (Nepf, 2012). The drag coefficient decreases downstream along the vegetation canopy because vegetation upstream shelter stems downstream from the impact velocity; thus, the bulk drag coefficient decreases downstream as vegetation stem density increases (Nepf, 1999; Nepf et al., 2007). However, no simple relations have been developed to date relating mean residence time to hydromorphic parameters. Predictive relationships may be derived for relative volumes of stream reaches occupied by emergent canopies. Thus, the mean residence time of emergent canopies is dependent on the following parameters:

$\tau=f\left(g, \Delta S, U_{\mathrm{c}}, d, a, C_{\mathrm{D}}\right)$.

Hypotheses relating the case-specific parameters of emergent aquatic vegetation to mean residence time are provided in Table 2.

\subsubsection{Submerged aquatic vegetation}

Submerged aquatic vegetation has a flow field characterized by the formation of a mixing layer at the canopy-water interface and a wake region in the canopy (Fig. 9; Finnigan, 2000; Ghisalberti and Nepf, 2002). The mean velocity profile in a submerged canopy is dependent on the canopy density and relative influence of canopy to bed drag (Wilson et al., 2003; Sukhodolov and Sukhodolova, 2006). In sparse submerged canopies, bed drag is larger than canopy drag and the mean velocity profile follows a turbulent logarithmic velocity profile, whereas in dense submerged canopies, bed drag is smaller than canopy drag and the mean velocity profile has an inflection point near the top of the canopy (Ghisalberti and Nepf, 2002; Wilson et al., 2003; Sukhodolov and Sukhodolova, 2006; Nepf and Ghisalberti, 2008). At the top of the canopy, the drag discontinuity increases velocity shear, causing flow separation and the formation of a mixing layer (Gambi et al., 1990; Stoesser et al., 2009). The inflection point in the velocity profile causes vortical structures in the mixing layer to billow and grow downstream due to Kelvin-Helmholtz instabilities (Ikeda and Kanazawa, 1996; Ghisalberti and Nepf, 2002; Nepf and Ghisalberti, 2008; Stoesser et al., 2009).

Canopy drag, density, and submergence depth - ratio of channel depth $\left(d_{\mathrm{C}}\right)$ to canopy height $(h)$ - determine the penetration depth of the mixing layer into the canopy (Fig. 9). Mixing layer penetration depth is inversely proportional to 
canopy drag and density and varies proportionally to submergence depth (Ghisalberti and Nepf, 2004; Poggi et al., 2004). For less dense and deeply submerged canopies $\left(d_{\mathrm{C}} / h>10\right)$, turbulent sweep and eject motions from vortical structures in the mixing layer penetrate the entire canopy height and transfer mass and momentum between the main channel and canopy (Fig. 9a; Fitzmaurice et al., 2004; Ghisalberti and Nepf, 2006; Nepf and Ghisalberti, 2008). Deeply submerged canopies promote sediment resuspension and transport. For dense and shallow submerged canopies $\left(d_{\mathrm{C}} / h \leq 5\right)$, turbulent sweep and eject motions from vortical structures in the mixing layer do not penetrate the entire canopy height (Fig. 9b; Ghisalberti and Nepf, 2004, 2005, 2009; Poggi et al., 2004). Shallow submerged canopies promote sediment entrainment by shielding the streambed from mixing layer turbulent stresses (Nepf, 2012). Entrainment and transport can be enhanced if the canopy is comprised of flexible plants and the main channel velocity exceeds a threshold value (Nepf, 2012). In this case, the advection of higher momentum-driven vortical structures causes a coherent waving pattern at the top of the canopy due to their downstream transport (Fig. 9c). This phenomenon is called a monami and causes canopy drag reduction and deeper penetration of vortical structures into the canopy (Ackerman and Okubo, 1993; Ghisalberti and Nepf, 2002, 2009; Nepf and Ghisalberti, 2008).

Nepf et al. (2007) developed a vertical transport model for flow through submerged aquatic canopies by dividing the canopy into an exchange and wake zone (Fig. 9c). Two timescales were developed, one each for the wake and exchange zone. The timescale of the wake zone, which is analogous to an STS zone, is given by (Nepf et al., 2007):

$T_{\text {wake }}=\frac{\left(h-\delta_{\mathrm{e}}\right)^{2}}{0.2 U_{\mathrm{c}} d}$,

where $\delta_{\mathrm{e}}$ is the mixing layer penetration depth into the canopy, [L]. For deeply submerged canopies, $\delta_{\mathrm{e}}=h$. For shallow submerged canopies, $\delta_{\mathrm{e}}<h$, and is predicted by (Nepf et al., 2007):

$\delta_{\mathrm{e}}=\frac{0.23 \pm 0.6}{0.6 a C_{\mathrm{D}}}$.

Vertical transport in the wake zone is governed by the hydraulic gradient and balances between bed and canopy drag (Ghisalberti and Nepf, 2009). Vertical transport in the exchange zone is governed by the hydraulic gradient and turbulent stresses (Nepf and Vivoni, 2000). The timescale for the exchange zone (mixing layer) is given by (Nepf et al., 2007):

$T_{\mathrm{e}}=\frac{\delta_{\mathrm{e}}}{E}=\frac{\delta_{\mathrm{e}}}{(0.17 \pm 0.02) \sqrt{-g S\left(d_{\mathrm{C}}-h\right)}\left(C_{\mathrm{D}} a h\right)^{0.13}}$,

where $E$ is the exchange velocity at the wake-exchange zone interface, $\left[\mathrm{L} \mathrm{T}^{-1}\right] ; g$ is the gravitational acceleration,
[ $\left.\mathrm{L} \mathrm{T}^{-2}\right] ; S$ is the channel slope; and $C_{\mathrm{D}}$ is the canopy drag coefficient given by $C_{\mathrm{D}}=2 g S d_{\mathrm{C}} / a h U_{\mathrm{c}}^{2}$. Note that Ghisalberti and Nepf (2005) found that there is a linear relationship between $E$ and the velocity difference between the main channel and wake region velocity, $\Delta U$ (Fig. 9c); therefore, the denominator in Eq. (12) can be approximated by $\Delta U$. Ghisalberti and Nepf (2005) also found that the vertical turbulent diffusivity can be approximated by $t_{\mathrm{ml}} \Delta U$, where $t_{\mathrm{ml}}$ is the total mixing layer thickness, [L]. From the vertical transport model, the mean residence time of a submerged canopy can be estimated by the wake zone timescale.

\subsection{Pools}

Pools are deep, slow-moving, recirculating in-stream flow structures and are prevalent in rivers and streams (Raven et al., 1998; Kang and Sotiropoulos, 2011). Pools typically form as part of step-pool or riffle-pool sequences (Montgomery and Buffington, 1997), adjacent to cascade reaches (Montgomery and Buffington, 1997), downstream of flow constrictions (Wright and Kaplinski, 2011), at abrupt changes in bed slope (Raven et al., 1998), or as backwater areas upstream and downstream of flow obstructions (Abbe and Montgomery, 2003). The flow dynamics of pools differ depending on the upstream flow conditions, whereby seasonal flow conditions can substantially change the flow field. Therefore, careful attention is needed when classifying the flow field of a pool.

\subsubsection{Pool type 1: the vertically submerged cavity}

In some riverine systems pools can be sufficiently deep, meaning that either an abrupt break in slope or deep scouring causes the pool bathymetry to be sufficiently below the bathymetry of the upstream reach at the pool head. In this case, the pool can be classified as a vertically submerged cavity. The flow dynamics of a vertically submerged cavity are analogous to the flow dynamics associated with lateral cavities (Fig. 10). Open channel flow across the top of the cavity causes flow separation at the upstream edge, mixing layer formation across the entire cavity entrance, and a recirculation region within the cavity. The cavity aspect ratio produces the same pattern of gyre dynamics observed in a lateral cavity. The predictive mean residence time in Eq. (1) can be applied to vertically submerged pools. Flume studies of flow past vertically submerged cavities show that the dynamics of mass and momentum exchange are the same for both lateral and vertically submerged cavities, where exponential RTDs arise and estimated entrainment coefficients are well within the range predicted for lateral cavities (e.g., Valentine and Wood, 1977; Seo and Maxwell, 1992; Chang et al., 2007). The predictive relationship in Eq. (2) was shown to work well for the experimental results of Seo and Maxwell (1992), who investigated exchange dynamics in a sequence of gravel-bed riffles with vertically-submerged pools. The relation holds 


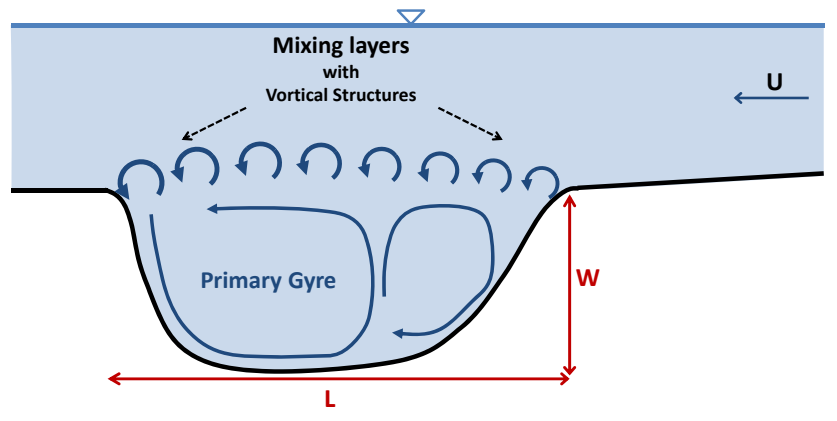

Fig. 10. Schematic of the flow field for a vertically submerged cavity. Gyre formation within the cavity is depicted for $W / L<0.5$. Note that gyre formation in vertically submerged cavities is equivalent to lateral cavities at the same $W / L$ (see Fig. 3).

when the cavity geometric parameters are redefined: $W$ is the mean distance from the mixing layer to the cavity streambed, $[\mathrm{L}] ; L$ is the cavity length (parallel to flow), [L]; $d_{\mathrm{E}}$ is the vertical length of the shear layer, [L]; and $d_{\text {STS }}$ is the cavity width (normal to flow), [L]. Table 2 provides a summary of qualitative relations between case-specific parameters and mean residence time.

\subsubsection{Pool type 2: the closed lateral cavity}

Many natural pools in streams have a geometry that resembles a lateral cavity, whereby one streambank is relatively straight and the other streambank has a lateral cutout due to erosion. At sufficiently high flows, the flow field will be characteristic to a lateral cavity (Fig. 11a). However, as the main channel flow decreases, the flow dynamics associated with lateral cavities can evolve into a flow field more characteristic of slower moving pools (Fig. 11b and c). For example, the mixing layer spanning the lateral cavity entrance will lose momentum as discharge decreases and the reattachment point at the downstream cavity edge will migrate upstream and reattach at a point along the wetted perimeter wall inside the cavity. As the reattachment point travels upstream due to lower channel velocities, flow will be advected directly into the cavity (Rathburn and Wohl, 2003). Thus, the mixing layer will not span the entire cavity length and a recirculation region will not encompass the entire cavity, as in an emergent lateral cavity (Shen and Floryan, 1985; Lawson and Barakos, 2011). The flow field will evolve from an emergent lateral cavity flow to a closed cavity flow. A closed cavity flow resembles a backward-facing step in the upstream cavity region and a forward-facing step in the downstream cavity region with no interaction between these flows (Fig. 11c). The mixing layer impinges at the streambank inside the cavity, reattaches to the streambank boundary, and a recirculation region forms in the upstream region of the cavity, forming a flow similar to that over a backward-facing step. The reattached boundary layer detaches in the downstream cavity region, forming a mixing layer that impinges on the trailing cavity edge and a recirculation region in the downstream cavity region, forming a flow similar to that over a forwardfacing step. If the reattachment point inside the cavity is sufficiently far downstream in the cavity, then a forward-facing step-like flow will not be observed (Fig. 11b) (e.g., Rathburn and Wohl, 2003; Wright and Kaplinski, 2011). Flow velocities decrease and disperse laterally toward the pool outlet within the main channel region adjacent to the closed cavity.

The closed cavity pool has a dynamic solute and sediment flushing pattern. Sediment entrainment occurs within the upstream and downstream recirculation regions of the closed cavity. At higher flows, the reattachment point in the cavity is located farther within the downstream cavity region. This causes a larger upstream recirculation region to form, increasing sediment entrainment in the upstream cavity region (Thompson, 1997; Thompson et al., 1998; Thompson and Hoffman, 1999). Sediment transport occurs in the downstream cavity region due to flow advection, causing scouring and erosion. At lower flows, the reattachment point migrates upstream, decreasing the size of the upstream recirculation region and promoting sediment transport. No work has been done to date to characterize the mean residence time of a closed lateral cavity.

The mean residence time of a closed lateral cavity is complex because it is dependent on parameters associated with an emergent lateral cavity, and backward- and forwardfacing steps. Mean residence time varies proportionally to $x_{\mathrm{FFS}}$ and $x_{\mathrm{BFS}}$, which are the lengths (parallel to flow) of the forward-facing and backward-facing steps, respectively. Mean residence time also varies proportionally to $d_{\mathrm{STS}, \mathrm{FFS}}$ and $d_{\mathrm{STS}, \mathrm{BFS}}$, which are the mean depths of the forwardfacing and backward-facing steps, respectively. Mean residence time varies inversely to $d_{\mathrm{E}, \mathrm{FFS}}$ and $d_{\mathrm{E}, \mathrm{BFS}}$, which are the mixing layer depths of the forward-facing and backwardfacing steps, respectively. An increase in the total cavity length (parallel to flow), $L$, and cavity width (normal to flow), $W$, increases the volume of fluid entrainment and mean residence time. In summary, the mean residence time of a closed lateral cavity is dependent on the following parameters (Table 2):

$\tau=f\left(u_{*}, U, d_{\mathrm{STS}, \mathrm{FFS}}, d_{\mathrm{STS}, \mathrm{BFS}}, d_{\mathrm{E}, \mathrm{FFS}}, d_{\mathrm{E}, \mathrm{BFS}}, v, g, W, L, x_{\mathrm{FFS}}, x_{\mathrm{BFS}}\right)$.

\subsubsection{Pool type 3: a recirculating reservoir}

Recirculating reservoir-type pools can form downstream of channel constrictions, cascades, riffles, log steps, or waterfalls. Deep, slow-moving, recirculating pools also can form upstream and downstream of wood debris that extend across the entire channel width and obstruct channel flow such that water overtops the wood debris. Wood debris accumulations can partially or completely impound channel flow. Specific types of wood debris include (1) stable orthogonal tree boles that form log steps; (2) combination debris jams - comprised of larger orthogonal in situ tree boles and smaller driftwood that grow laterally from upstream debris accumulations and 


\section{Plan View}

A)

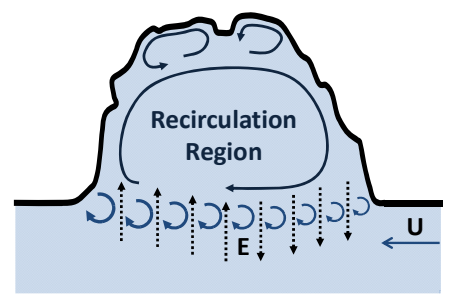

Emergent Lateral Cavity
B)

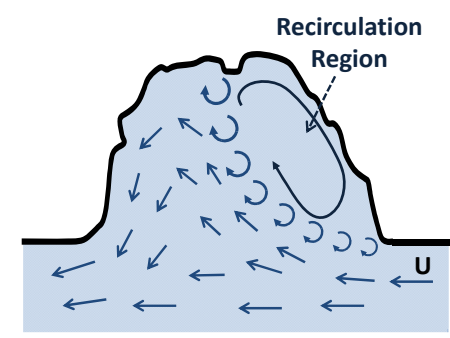

Transition
C)

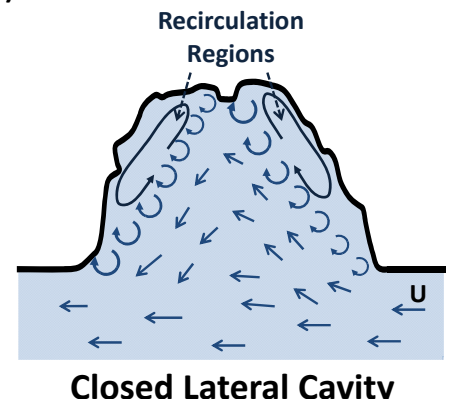

Fig. 11. Schematics showing the evolution of the flow field from an emergent lateral cavity (A) to a closed lateral cavity (C).

have a larger lateral extent than the channel width; (3) chaotic accumulations of waterborne driftwood; and (4) beaver dams (Abbe and Montgomery, 2003).

The mean residence time of a reservoir-type pool can be obtained from Eq. (1) by redefining hydromorphic parameters: $W$ and $L$ are the pool width (normal to flow) and length (parallel to flow), respectively, [L]; $d_{\mathrm{STS}}$ is the mean pool depth, [L]; $d_{\mathrm{E}}$ is the mean pool depth at the pool outlet, [L]; and $E$ is the exchange velocity leaving the pool through the pool outlet, [ $\left[\mathrm{L} \mathrm{T}^{-1}\right]$. An increase in $W, L$, or $d_{\mathrm{STS}}$ increases mean residence time, whereas an increase in $d_{\mathrm{E}}$ decreases mean residence time. An increase in main channel velocity increases gyre circulation of the recirculating pool, which decreases mean residence time. As dams and jams typically form recirculating pools, the relative porosity, $\theta$, of the wood debris attributed to underflow and flow through the debris also should be considered, which decreases mean residence time. The mean residence time of a reservoir-type pool can be obtained if either the exchange velocity is known, or an entrainment coefficient can be substituted into the second relation in Eq. (1). No work to date has estimated an entrainment coefficient for this type of flow; however, the mean residence time of recirculating reservoir-type pools is dependent on the following parameters (Table 2):

$\tau=f\left(u_{*}, U, d_{\mathrm{STS}}, d_{\mathrm{E}}, v, g, W, L, \theta\right)$.

\section{Recirculating reservoir: a jet-like flow}

A recirculating reservoir-type pool downstream of channel constrictions, cascades, or riffles forms when faster main channel velocities upstream of the pool converge with slower flow downstream, transferring momentum and driving flow recirculation. Higher flow velocities enter the pool head as jet-like flows, causing flow separation and, sometimes, the formation of localized recirculation regions (Fig. 13a; Peterson and Mohanty, 1960; Bathurst, 1979; Thompson et al., 1996; Raven et al., 1998; Kang and Sotiropoulos, 2011). The localized recirculation regions are dominated by one large gyre and form to the left and right banks of the incoming jet-like flow at the pool head (Kang and Sotiropoulos, 2011). Momentum exchange of the recirculating gyres with the incoming jet can increase the incoming flow velocities at the pool head (Booker et al., 2001). As the higher incoming velocities encounter slower moving water downstream in the pool, the velocity decreases and flow disperses laterally toward the pool outlet (Sear, 1996; Kang and Sotiropoulos, 2011). Decelerating flow velocities toward the pool outlet cause sediment deposition (Abbe and Montgomery, 1996). These pools typically have graded beds where coarser sediments are deposited near the pool head due to higher flows and scouring, and finer sediments are deposited near the pool outlet as flow decreases longitudinally through the pool (Iseya and Ikeda, 1987; Lisle et al., 1991).

\section{Recirculating reservoir: flow impingement}

A recirculating reservoir-type pool upstream of wood debris (e.g., beaver dams, log jams) forms when flow impingement deflects flow laterally and downward toward the streambed. The accelerated downwelling flow impinges on the streambed, causing scouring and erosion (Abbe and Montgomery, 1996). The downwelling flow forms a scour pool just upstream of the wood debris (see Fig. 1b for example). Sweeping motions in and out of the scour hole generate a recirculating flow deep in the pool. The laterally deflected flow scours and erodes the streambed on either side of the wood debris, and some of the flow is deflected upstream in a strong backward flow toward the oncoming streamflow (Fig. 12b). The balance of incoming and backward flow forces creates a stagnation point upstream of the debris and causes flow recirculation. For pools formed upstream of wood debris, sand bars can develop just upstream of the scour pool beneath the stagnation point as a result of flow deceleration and deposition (Abbe and Montgomery, 2003). 

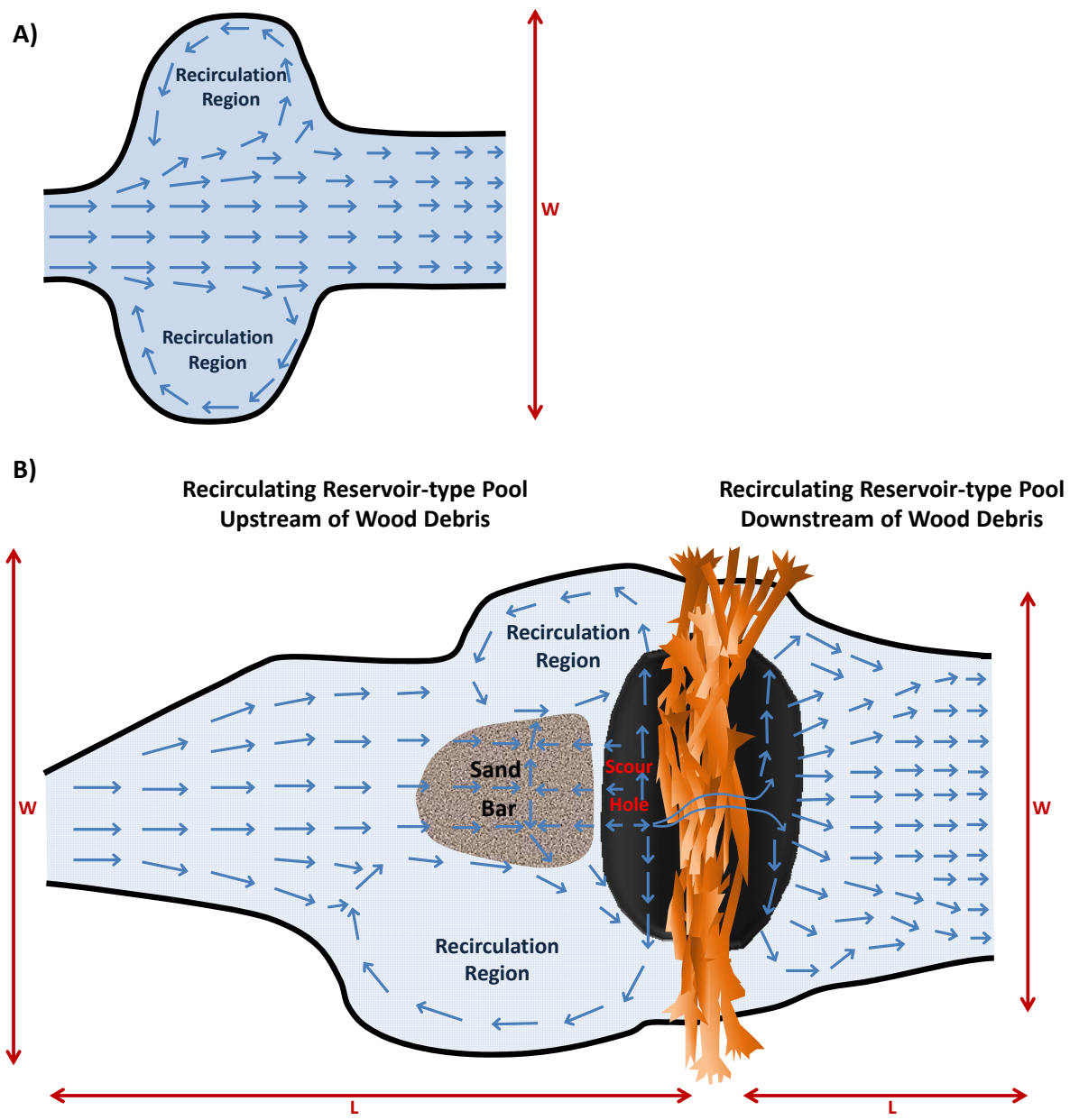

Fig. 12. Schematics illustrating three different flow field examples of recirculating reservoir-type pools. (A) A jet-like flow forms recirculation regions on each side of the pool near the entrance. (B) (left-hand side) Upstream of in-channel obstacle, flow impingement forms recirculation regions near streambanks and a deep recirculating flow in scour hole. (B) (right-hand side) Downstream of in-channel obstacle, vertical jetlike flow enters pools and forms deep recirculating flow in scour hole.

\section{Recirculating reservoir: scour pool}

A recirculating reservoir-type pool downstream of a waterfall, log step, debris dam, or log jam is typically called a scour or plunge pool (Lamb et al., 2007). In this case, the jet of upstream flow enters vertically at the pool head and drives downwelling flow (and falling sediments) toward the streambed (Fig. 12b). A scour hole forms at the pool head due to downwelling flow scouring the streambed. For rapidly varied flow, the incoming jet-like flow can form a hydraulic jump comprised of streamwise-oriented rollers (Endreny et al., 2011). The flow velocity in the pool decreases and disperses laterally toward the outlet.

\subsection{Meander bends}

Meander bends are characterized by a complex interaction of turbulent flow structures. Planform longitudinal bank curvature causes flow separation along the inner and outer banks and an imbalance of centrifugal and transverse pressure forces, inducing the direction of near-surface flow toward the outer bank (cut bank) and near-streambed flow toward the inner bank (point bar) (Fig. 13; Van Bendegom, 1947; Rozovskii, 1957; Bagnold, 1960; Leopold et al., 1960; Dietrich and Smith, 1983; Thompson, 1986). Flow separation at the start of the bend forms two mixing layers: one along the inner bank and one along the outer bank nearly across from one another. A strong jet-like flow forms between the mixing layers and impinges near the apex of the outer bank, raising water surface levels and inducing downwelling flow toward the streambed (Blanckaert, 2010). Downwelling flow impingement and subsequent transverse deflection toward the inner bank, which is termed a curvature-induced secondary flow, is the predominant cause of bed scouring along the outer bank (Thomson, 1876; Johannesson and Parker, 1989; Hodskinson and Ferguson, 1998; Blanckaert, 2010). Scouring is also influenced by 


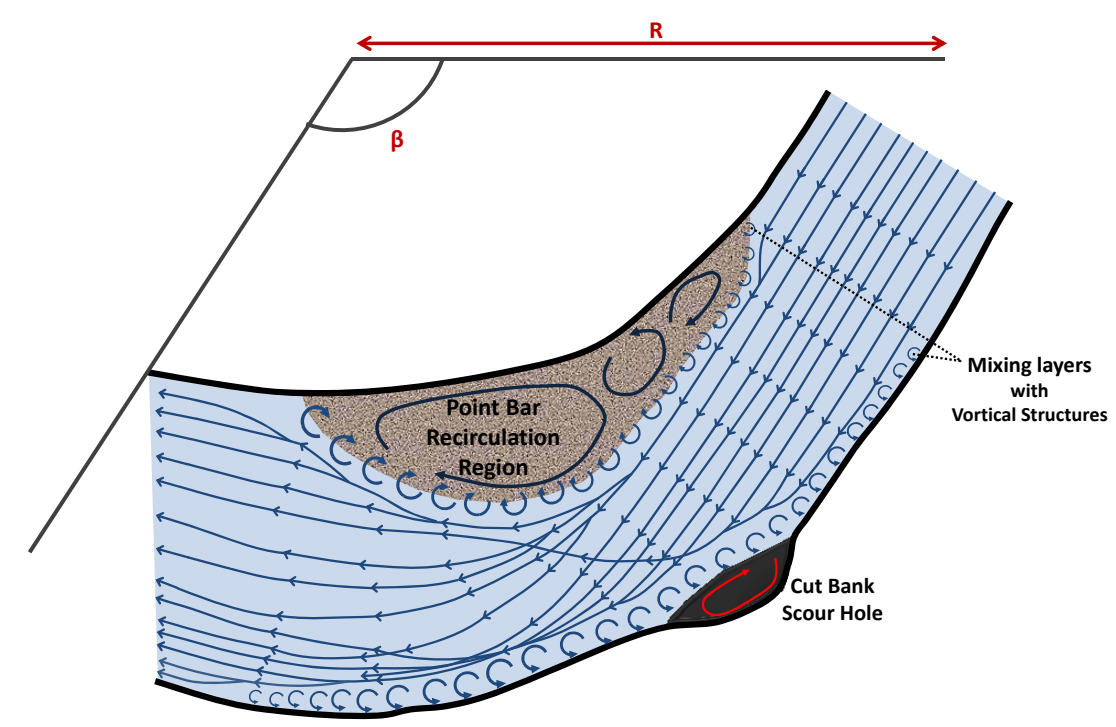

Fig. 13. Schematic of the flow field for a meander bend. (Figure adapted from the work of Kang and Sotiropoulos, 2011.)

small vortices that form adjacent to the outer bank when centrifugal forces interact with streamwise anisotropic vortical structures in the outer bank mixing layer (Hey and Thorne, 1975; Bathurst et al., 1979; Thorne et al., 1985; Kang and Sotiropoulos, 2011). Jamieson et al. (2010) showed that small outer bank vortices are centered above scour holes. Increased transverse Reynolds stresses and turbulent kinetic energy are the mechanisms by which curvature-induced secondary flow and small outer bank gyres scour and erode the outer bank (Blanckaert and Graf, 2001; Blanckaert and de Vriend, 2004; van Balen et al., 2009).

The flow dynamics associated with the inner bank induce solute and sediment transport and entrainment. The inner bank mixing layer advects vortical structures downstream (Fig. 13). The vortical structures typically impinge just downstream of the meander bend's point of highest curvature and cause the unsteady transport of vorticity and momentum toward the inner bank, forming a recirculation region (Bagnold, 1960; Leeder and Bridges, 1975; Kang and Sotiropoulos, 2011). The inner bank recirculation region is comprised of a large primary gyre centered atop the point bar in the region of high bend curvature. A number of smaller secondary gyres develop upstream along the point bar due to primary gyre momentum exchange (Leeder and Bridges, 1975; Ferguson et al., 2003). Deposition and entrainment occur along the inner bank because inner bank gyres have low velocities and turbulent mixing (i.e., low turbulent kinetic energy) (Schmidt, 1990; Kang and Sotiropoulos, 2011). Predominant locations of sediment entrainment within the inner bank recirculation region occur near the primary gyre vortex center and the separation and reattachment points (Rubin et al., 1990; Schmidt, 1990). Kang and Sotiropoulos (2011) found that the primary gyre vortex center is situated directly atop the apex of the point bar.
Relations have been found in the hydrology literature to relate hydromorphic parameters in a meander bend. Leeder and Bridges (1975) and Schmidt (1990) found that flow separation and vortical structure coherence, which influence inner bank recirculation and entrainment, is dependent on the Froude number and the degree of meander bend curvature. The meander bend curvature is quantified using a curvature ratio, $R / B$, where $R$ is the meander bend radius of curvature, $[\mathrm{L}]$; and $B$ is the main channel width, $[\mathrm{L}]$ (Leeder and Bridges, 1975; Blanckaert and de Vriend, 2010). A hydromorphic parameter for sharply curved meander bends is given by $C_{\mathrm{f}}^{-1} d_{\mathrm{C}} / B$, where $C_{\mathrm{f}}$ is the Chézy-type friction coefficient; and $d_{\mathrm{C}}$ is the channel depth, [L] (Blanckaert and de Vriend, 2010). Blanckaert (2010) showed that the flow dynamics of the outer and inner bank are strongly influenced by bed topography, where the inner bank recirculation region is dependent on the inner bank mixing layer depth, $d_{\mathrm{E}}$. Blanckaert (2010) also derived a required condition for bank curvature-induced flow separation and recirculation: $R_{\min } / B<0.5\left(C_{\mathrm{f}}^{-1} d_{\mathrm{C}} / B\right)^{1 / 2}$, where $R_{\min }$ is the minimum radius of curvature, [L].

No relations have been developed to date to characterize the mean residence time of solutes and sediments entrained by a meander bend. Qualitatively, the mean residence time of the inner bank recirculation region varies proportionally to point bar width (normal to flow), $W$, and point bar length (parallel to flow), $L$. An increase in $W$ increases the size of gyres in the recirculation region and an increase in $L$ increases the number of secondary gyres. Point bar length and mean residence time increase as the meander bend radius of curvature increases. The minimum radius of curvature is associated with the smallest point bar that can form along a meander bend, which will decrease mean residence time. Larger main channel widths form larger point bar widths, which 

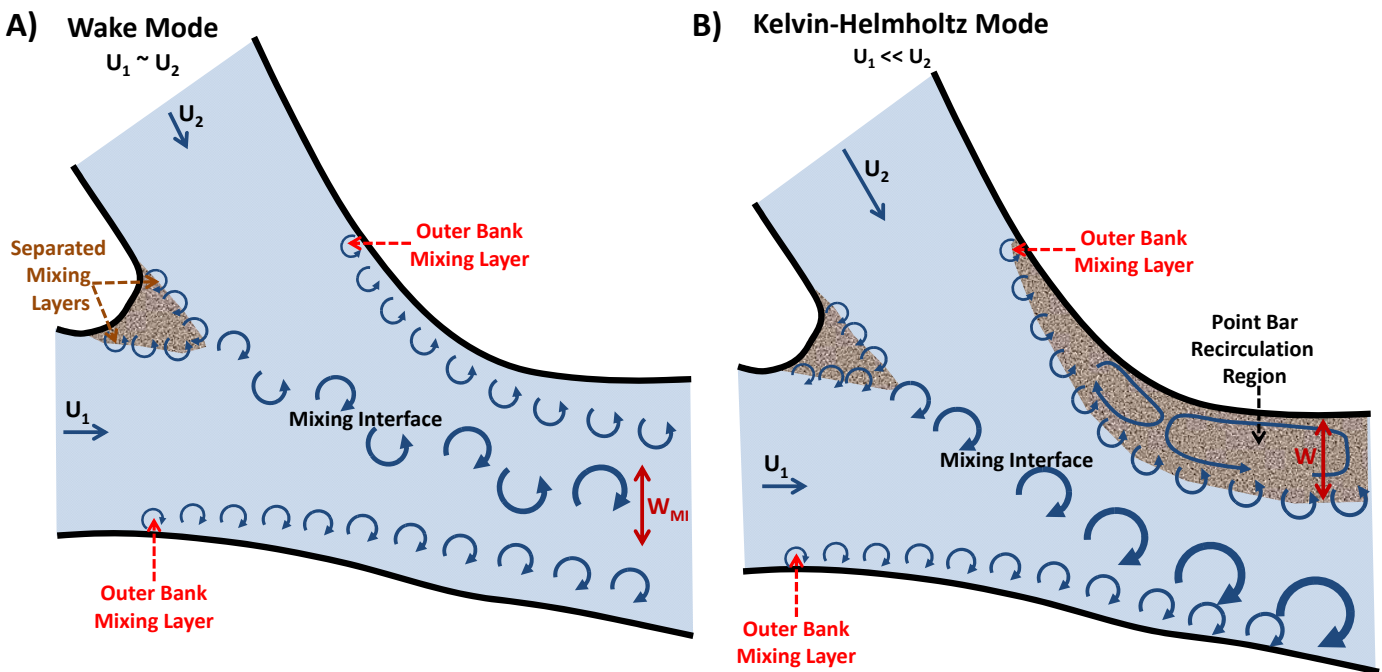

Fig. 14. Flow field schematics of a stream confluence with a small velocity ratio between converging streams (A) and with a large velocity ratio between converging streams (B). (Figure adapted from the work of Miyawaki et al., 2010 and Constantinescu et al., 2011.)

increases mean residence time. An increase in mean point bar depth, $d_{\mathrm{STS}}$, increases the volume of solute entrainment and mean residence time, whereas an increase in mean inner bank mixing layer depth, $d_{\mathrm{E}}$, increases mass and momentum exchange across the mixing layer, which decreases mean residence time. Frictional forces quantified by the Chézy-type friction coefficient and kinematic viscosity increase mean residence time. Main channel velocity and gravitational acceleration drive momentum exchange across the mixing layer and decrease mean residence time. In summary, the mean residence time of a meander bend point bar is dependent on the following parameters (see Table 2):

$\tau=f\left(U, d_{\mathrm{STS}}, d_{\mathrm{E}}, v, g, R, R_{\min }, B, C_{\mathrm{f}}, W, L\right)$.

\subsection{Confluence of streams}

The flow field at the confluence of two non-parallel streams is characterized by flow separation at the point of streambank convergence (junction point) and the development of a mixing interface downstream of the junction point comprised of streamwise-oriented vortical structures (Fig. 14; Rhoads and Kenworthy, 1995; Bradbrook et al., 1998). The streamwiseoriented vortical structures are formed by the higher transverse momentum generated from incoming stream convergence (Sukhodolov and Rhoads, 2001). Vortical structures in the free mixing interface are shed from each stream at the junction point and advect downstream, accelerating the downstream flow within the mixing interface (Rhoads and Kenworthy, 1995). Vortical structure pairing has been documented in the mixing interface, whereby shedded vortical structures from each stream are advected downstream in pairs from the junction point (e.g., Rhoads and Kenworthy, 1998; Rhoads and Sukhodolov, 2001; Constantinescu et al., 2011).
The pattern of vortical structure pairing in the mixing interface has been described as being in the wake, Kelvin-Helmholtz, or combined wake-Kelvin-Helmholtz mode (Fig. 14; Miyawaki et al., 2009, 2010; Constantinescu et al., 2011). When the large-scale streamwise-oriented vortical structures rotate in opposite directions (counter-rotating), the mixing interface is in the wake mode (Fig. 14a), whereas when the large-scale vortical structures rotate in the same direction (co-rotating), the mixing interface is in the KelvinHelmholtz mode (Fig. 14b; Constantinescu et al., 2011). In the wake mode, the counter-rotating vortices are shed from the separation point and grow downstream (from weak Kelvin-Helmholtz instabilities) without an increase to their circulation, which limits their ability to entrain sediment. In the Kelvin-Helmholtz mode, strong Kelvin-Helmholtz instabilities arise in the mixing interface due to interactions between vortices in the mixing layers, causing vortex pairing between the mixing layers (Constantinescu et al., 2011). In this case, the mixing interface is comprised of vortices that merge, grow, and increase their circulation as they are advected downstream from the separation point, which increases their ability to entrain sediment. In some cases, such as high velocity ratios and confluence angles $(\beta)$ between two converging streams, the mixing interface can have vortical structures whose interactions can be characterized by both the Kelvin-Helmholtz and wake mode (Miyawaki et al., 2010).

Mixing interface position, mode, and coherence strength is dependent on the velocity (or momentum) ratio of the incoming streams, bathymetry (i.e., bed discordances), and the confluence angle at the junction point (Biron et al., 1996a,b). Note that the velocity ratio is given by $U_{1} / U_{2}$, where $U_{1}$ and $U_{2}$ are the mean channel velocities of the main and tributary channels, respectively, $\left[\mathrm{L} \mathrm{T}^{-1}\right]$. If the velocity ratio 
between the incoming streams is small, then the mixing interface is in the wake mode (Fig. 14a). The mixing interface is comprised of strongly coherent vortical structures and neither stream pushes the mixing interface closer to the opposite streambank (i.e., the mixing interface is centered downstream of the point of stream convergence) (Constantinescu et al., 2011). However, if the velocity ratio between the incoming streams is large, then the mixing interface is in the Kelvin-Helmholtz mode and the stream with the larger momentum pushes the mixing layer interface toward the opposite streambank (Fig. 14b; Miyawaki et al., 2010). Vortical structures in the mixing layer of the higher momentum stream have greater coherence than vortical structures in the mixing layer of the lower momentum stream (Rhoads and Kenworthy, 1998). The coherence of vortical structures in the mixing interface also depends on the stream-bed topography and the confluence angle of the incoming streams, where concordant streambeds and larger confluence angles generally increase vortical structure coherence (Miyawaki et al., 2010).

An additional flow field complexity is the separated mixing layers that form along the outer streambanks of the incoming streams at the confluence. The longitudinal bank curvature imposed by the confluence causes outer streambank flow separation. At high confluence angles and low momentum ratios, flow separation at the outer streambanks forms mixing layers that interact with the mixing interface (Fig. 14a). At high confluence angles and momentum ratios, the higher momentum stream pushes the mixing interface toward the opposite streambank and the lower momentum stream mixing layer interacts with the mixing interface (Fig. 14b). The interaction of vortical structures causes scouring and erosion. Flow separation, mixing layer formation and downstream impingement at the outer bank of the higher momentum stream is the same as that of a meander bend. A recirculation region forms along the outer bank (near the high momentum stream) atop a point bar (Fig. 14b; Constantinescu et al., 2011).

The mean residence time of solutes and sediments in stream confluences has not been studied to date. Key parameters influencing entrainment in the mixing interface are the confluence angle, velocity and momentum ratios, and bed concordance. Larger confluence angles $(\beta)$ and higher momentum ratios $\left(U_{1} / U_{2}\right)$ increase solute entrainment and mean residence time. The exact influence of bed topography discordance on vortical structure coherence and transport is still largely unknown as discordant topographic features between channels have been found to both increase vortical structure coherence (e.g., Miyawaki et al., 2010) and decrease vortical structure coherence (e.g., De Serres et al., 1999). An increase in mean width $\left(W_{\mathrm{MI}}\right)$ and depth $\left(d_{\mathrm{MI}}\right)$ of the mixing interface increase the volume of solute entrainment and mean residence time. Main channel width downstream of the confluence, $B$, increases the potential for mixing interface spreading $\left(W_{\mathrm{MI}}\right)$ and mean residence time.
Frictional forces quantified by the Chézy-type friction coefficient and kinematic viscosity increase mean residence time. Thus, the mean residence time of a stream confluence (without the formation of a point bar) is dependent on the following parameters:

$\tau=f\left(C_{\mathrm{f}}, U_{1}, U_{2}, W_{\mathrm{MI}}, \beta, d_{\mathrm{MI}}, v, g, B\right)$.

If a point bar is present, the mean residence time is also dependent on parameters associated with a meander bend (see Table 2):

$\tau=f\left(C_{\mathrm{f}}, U_{1}, U_{2}, W_{\mathrm{MI}}, \beta, d_{\mathrm{MI}}, v, g, B, d_{\mathrm{E}}, d_{\mathrm{STS}}, R, R_{\min }, W, L\right)$.

\section{Utilizing the STS classification scheme}

\subsection{Development of predictive mean residence time relationships}

We hypothesize that, for each STS type (and subtype) identified in the classification scheme, mean residence time relationships can be derived for a range of flow conditions and geometries using field-measurable hydromorphic parameters. Employing dimensional analysis, nondimensional mean residence times can be related to a combination of nondimensional quantities, such as Reynolds number, Froude number, shape factors, bed roughness parameters, aspect ratios, submergence ratios, discharge ratios, and other case-specific parameters (see Table 3). Nondimensional mean residence times can then be compared to collected data for verification. This method was recently utilized by Jackson et al. (2013) to successfully relate the nondimensional mean residence time of a lateral emergent cavity to six nondimensional quantities. Nepf et al. (2007) also developed residence time relationships by dividing a submerged aquatic canopy into two zones: an exchange and wake zone. The exchange zone timescale (Eq. 10) is representative of solute residence time in the mixing layer interface and the wake zone timescale (Eq. 12) is representative of solute residence time in the canopy (STS). Thus, in some cases, mean residence time relationships may need to be derived for both the STS and the mixing layer interface if solute exchange is not a rapid process and solute becomes entrained in the mixing layer at timescales near the order of the STS residence timescale.

For each STS identified, mean residence time is a function of a number of hydromorphic parameters (see Eqs. 1-17). In all cases, mean residence time is dependent on the main channel velocity, gravitational acceleration, and bed friction (or canopy drag for aquatic vegetation) (Table 3). These parameters form three nondimensional quantities: Reynolds number, Froude number, and a bed roughness factor; however, the characteristic length scale differs among STS types. For example, the mean depth at the mixing layer interface $\left(d_{\mathrm{E}}\right)$ is the characteristic length scale for the emergent lateral cavity, whereas the stem diameter $(d)$ is the characteristic 
Table 3. Summary of hydromorphic parameters characteristic to STS types.

\begin{tabular}{|c|c|c|c|c|}
\hline STS type & Flow & Roughness & Constants & Case-specific parameters \\
\hline \multicolumn{5}{|l|}{ Lateral cavity } \\
\hline Emergent & $U$ & $u_{*}$ & $g v$ & $d_{\mathrm{E}}, d_{\mathrm{STS}}, W, L$ \\
\hline Submerged & $U$ & $u_{*}$ & $g v$ & $d_{\mathrm{E}}, d_{\mathrm{STS}}, W, L,\left(d_{\mathrm{C}}-d_{\mathrm{STS}}\right)$ \\
\hline \multicolumn{5}{|l|}{ Protruding flow obstructions } \\
\hline Backward-facing step & $U$ & $u_{*}$ & $g v$ & $d_{\mathrm{E}, \mathrm{BFS}}, d_{\mathrm{STS}, \mathrm{BFS}}, W, x_{\mathrm{BFS}}, \gamma$ \\
\hline Forward-facing step & $U$ & $u_{*}$ & $g v$ & $d_{\mathrm{E}, \mathrm{FFS}}, d_{\mathrm{E}, \mathrm{BFS}}, d_{\mathrm{STS}, \mathrm{FFS}}, d_{\mathrm{STS}, \mathrm{BFS}}, w, x_{\mathrm{BFS}}, x_{\mathrm{FFS}}, y_{\mathrm{FFS}}, \gamma$ \\
\hline \multicolumn{5}{|l|}{ Isolated flow obstructions } \\
\hline Emergent & $U$ & $u *$ & $g v$ & $d_{\mathrm{E}}, d_{\mathrm{STS}}, W, B, \theta$ \\
\hline Submerged & $U$ & $u_{*}$ & $g v$ & $d_{\mathrm{E}}, d_{\mathrm{STS}}, W, B, \theta,\left(d_{\mathrm{C}}-d_{\mathrm{STS}}\right)$ \\
\hline Cascades and riffles & $U$ & $u_{*}$ & $g v$ & $d_{\mathrm{C}}, D_{\mathrm{g}}, H_{\mathrm{g}}, L_{\mathrm{R}}, S_{\mathrm{g}},\left(d_{\mathrm{C}}-H_{\mathrm{g}}\right)$ \\
\hline \multicolumn{5}{|l|}{ Aquatic vegetation } \\
\hline Emergent & $U_{\mathrm{c}}$ & $C_{\mathrm{D}}$ & $g$ & $a, d, \Delta S$ \\
\hline Submerged & $U_{\mathrm{c}}$ & $C_{\mathrm{D}}$ & $g$ & $a, d, h, d_{\mathrm{C}}, \delta_{\mathrm{e}}, S, \Delta U$ \\
\hline \multicolumn{5}{|l|}{ Pools } \\
\hline Vertically submerged cavity & $U$ & $u_{*}$ & $g v$ & $d_{\mathrm{E}}, d_{\mathrm{STS}}, W, L$ \\
\hline Closed lateral cavity & $U$ & $u_{*}$ & $g \nu$ & $d_{\mathrm{E}, \mathrm{FFS}}, d_{\mathrm{E}, \mathrm{BFS}}, W, L, d_{\mathrm{STS}, \mathrm{FFS}}, d_{\mathrm{STS}, \mathrm{BFS}}, x_{\mathrm{FFS}}, x_{\mathrm{BFS}}$ \\
\hline Recirculating reservoir & $U$ & $u_{*}$ & $g v$ & $d_{\mathrm{E}}, d_{\mathrm{STS}}, W, L, \theta$ \\
\hline Meander bends & $U$ & $C_{\mathrm{f}}$ & $g v$ & $d_{\mathrm{E}}, d_{\mathrm{STS}}, W, L, B, R, R_{\mathrm{min}}$ \\
\hline Confluence of streams & $U_{1}, U_{2}$ & $C_{\mathrm{f}}$ & $g v$ & $d_{\mathrm{E}}, d_{\mathrm{STS}}, d_{\mathrm{MI}}, W, L, W_{\mathrm{MI}}, B, \beta, R, R_{\min }$ \\
\hline
\end{tabular}

length scale for aquatic vegetation. In most cases the characteristic length scale has not been identified. The definition of bed roughness will also be defined differently between cases. In emergent lateral cavities, the channel bed roughness of the upstream boundary layer (i.e., upstream of the cavity separation point) is used to define the shear velocity. For the confluence of two streams, the bed roughness of each stream above the confluence must be considered to define the shear velocity. Thus, case-specific hydromorphic parameters dictate the flow structure and mean residence time characteristic to each STS, and detailed analyses are needed to determine appropriate metrics for defining hydraulic, geometric, and roughness quantities. As a starting point, we provide qualitative relations between mean residence time and case-specific hydraulic and morphologic parameters in Table 2 for each STS type.

\subsection{Application of STS classification}

To implement the STS classification scheme and quantitatively separate STS from HTS in a tracer test, we propose a method that deconvolves the STS residence time distribution (RTD) from the total transient storage RTD to obtain the HTS RTD. Implementation of this proposal is well beyond the scope of this manuscript, and so we only provide a brief overview. We can think of the total transient storage RTD as closely related to the late-time tailing behavior of the main channel tracer concentration breakthrough curve (BTC) in a reach-scale tracer test (Haggerty et al., 2000). Transient storage increases tracer mean residence time along a channel reach, where longer mean residence times increase the skewness (tailing) of the main channel concentration BTC. This method extends the work of Haggerty et al. (2002), who performed a reach-scale tracer injection to obtain tracer concentration BTCs in a high-gradient (2nd-order) stream. A multirate mass transfer (MRMT) model with a late-time power-law RTD was used to characterize the late-time behavior (tailing) of the measured BTC and estimate the HTS RTD. Note that the choice of transient storage model is dependent on the type of RTD measured in a reach-scale tracer test. The slope of the best-fit line through the late-time tailing of the measured BTC is used in conjunction with the harmonic mean approximation to the RTD to obtain the total transient storage RTD (see Table 1 of Haggerty et al., 2000, for a listing of different RTDs and corresponding harmonic mean approximations). STS mean residence times can then be deconvolved to obtain the HTS RTD. Once the type of RTD is determined and a transient storage model is selected, an effective STS mean residence time and an estimate of the reach-scale STS volume fraction ( $V_{\mathrm{STS}}$ ) can be used for model parameter estimation.

We highlight that a number of transient storage models have been developed with similar conceptual frameworks but differ in the type of RTD assumed. All of these models could be applied in a similar fashion to that outlined above, with minor modifications. The OTIS model (Bencala 
and Walters, 1983; Runkel, 1998) assumes an exponential RTD based on a perfectly mixed continuous stirred tank reactor with a single-rate mass transfer coefficient. Choi et al. (2000) modified the OTIS model to formulate a twozone transient storage model that represents STS and HTS separately with exponential RTDs. Wörman et al. (2002) developed the Advective-Storage-Path (ASP) model by rederiving the transient storage equations to include moment methods for a lognormal RTD. The continuous time random walk (CTRW) model (Montroll and Weiss, 1965; Berkowitz and Scher, 1998; Boano et al., 2007; Berkowitz et al., 2008) could be used in a fashion similar to the MRMT model by employing separate RTDs for HTS and STS. An application of the CTRW model by Stonedahl et al. (2012) could possibly be extended to do this task.

\section{Advantages and limitations of STS classification}

A fluid-mechanics based STS classification scheme will provide a number of advantages when implemented in transient storage models. First, predictive relationships will be developed based on a small number of field-measurable hydraulic and morphologic parameters. Second, the morphologic parameters will provide a more accurate estimate of the reachscale STS volume fraction ( $\left.V_{\mathrm{STS}}\right)$. Third, an accurate effective STS mean residence time can be obtained from the predictive relationships $\left(\tau_{\mathrm{STS}}\right)$. Fourth, an accurate estimate of the HTS mean residence time ( $\tau_{\mathrm{HTS}}$ ) can be obtained by deconvolving the late-time tailing of the total transient storage RTD. Fifth, effective STS and HTS mass exchange rate coefficients can be obtained from the inverse of the effective STS and HTS mean residence times, respectively. Therefore, all transient storage parameters will be constrained during parameter optimization with the exception of the longitudinal dispersion coefficient. Sixth, the predictive mean residence time relationships will be developed based on the underlying physics driving mass and momentum exchange; thus, these relationships will be process-based and will more accurately describe residence time dynamics. Lastly, the predictive relationships will allow for a priori estimates of STS mean residence times that will reduce extensive data requirements currently in practice. For example, in reach-scale tracer tests, additional sensors are emplaced in individual STS zones (e.g., Gooseff et al., 2008; O'Connor et al., 2010) as well as in the main channel adjacent to STS zones to obtain STS RTDs (e.g., Gooseff et al., 2011). The predictive relationships will eliminate the need for emplacing additional sensors in individual STS zones to obtain STS RTDs.

One complication that can arise when using the fluidmechanics based STS classification scheme is the identification of STS zones that may not precisely fit into the STS types presented. The STS classification scheme identifies and describes the most predominant types of STS in fluvial systems. Some STS may have flow structure characteristics that deviate somewhat from the flow structure described in the classification. For example, one may find an emergent lateral cavity in a channel with a slightly permeable upstream boundary, such as if wood debris upstream of the cavity forms the upstream boundary. In the case that the amount of leakage through the wood debris does not significantly disrupt the flow structure (i.e., the recirculation region is pronounced), then the leakage can be assumed negligible. However, in the case that the amount of leakage through the wood debris does significantly disrupt the flow structure (i.e., flow in the upstream cavity region is disrupted while a recirculation region forms in the downstream region), then the leakage cannot be neglected. For these unique cases sensors may need to be placed in the individual STS zones and in the main channel adjacent to the STS to deconvolve the STS RTD, using the method employed by Gooseff et al. (2011), to obtain the STS mean residence time.

\section{Broader impacts of STS classification scheme}

The STS classification scheme presented provides a foundation for future studies in the areas of fluid dynamics, geomorphology and hydrology. In fluid dynamics, the classification scheme has presented flow types where little to no work has been done, such as submerged backward- and forward-facing steps. In addition, flow types where more work needs to be done are highlighted. Investigations into the flow structure of different STS types (and the influence of different hydromorphic parameters on the mean flow field) will aid in the development of predictive mean residence time relationships.

In geomorphology, the fluid-mechanics based classification scheme may remove complexities and ambiguities associated with relating different types of STS between different riverine systems. For example, backwater areas in riverine systems are typically defined as pools; however, lateral cavities, backward- and forward-facing steps, vertically submerged cavities, closed cavities, and recirculating reservoirs have all been identified as pools in the hydrology literature. This has led to complications and inconsistencies in the study of pool maintenance and sediment transport dynamics. As an example, in the well-studied riffle-pool sequence, many hydraulic reversal theories have been provided in the literature to describe the maintenance of pools based on hydraulic mechanisms, including an abrupt decrease in water surface slope (Keller, 1971); a decrease in mean cross-sectional velocity (Lane and Borland, 1954); and changes in near-bed velocity and shear stress (Keller, 1969, 1971; Lisle, 1979). These hydraulic reversal theories postulate that high velocities and bed shear stress occur on the upstream riffles, and low velocities and bed shear stress occur in the downstream pools at low flows, causing the downstream transport of fine sediment into pools (Hack, 1957; Keller, 1971; Lisle and Hilton, 1992). Conversely, at high flows, lower velocities and bed shear stress are observed on the riffles compared 
to the mid-pool region, causing the downstream transport of coarse sediment from the pools to downstream riffles (Keller, 1971; Andrews, 1979; Lisle, 1979; Ashworth, 1987; Clifford, 1993). Booker et al. (2001) showed that hydraulic reversal theories weakly explain pool formation dynamics because decreased riffle velocity and bed shear stress were responsible for the maintenance of three out of eight pools that form in riffle-pool sequences along a meander bend of the Highland Water in the UK. Furthermore, Campbell and Sidle (1985), Sidle (1988), Carling (1991), Keller and Florsheim (1993), and Sear (1996) also observed weak relationships between hydraulic reversals along upstream riffles and maintenance of their downstream pools at higher discharges, suggesting that these hydraulic reversals are not the main mechanism for pool formation and maintenance. We postulate another explanation: the flow dynamics of exchange may change, producing a different type of flow field in the pool at high discharges, such as from a closed cavity to a recirculating reservoir. Therefore, the hydraulic reversal theory may only be applicable to pools with a specific flow structure.

In hydrology, developing predictive mean residence times for different STS using field-measurable parameters will provide an accurate, reliable, and inexpensive method for estimating STS. This approach will allow for the quantitative separation of STS from HTS in a tracer test, where STS mean residence times can be deconvolved from the total transient storage mean residence time to obtain the HTS RTD. Quantitatively separating STS from HTS will allow for the determination of whether specific processes are occurring instream, in the hyporheic zone, or both (Harvey and Wagner, 2000). Predictive relationships will also provide a more direct comparison between transient storage and solute dynamics and exchange processes among morphologically diverse streams (D'Angelo et al., 1993). Furthermore, predictive relationships will aid stream restoration efforts in mitigating nutrient transport by providing a quantitative means for assessing the impact of hydromorphic parameters on in-stream structure design (Baker et al., 2012). For example, stream restoration projects typically restore stream ecosystems by emplacing in-stream structures that increase biological diversity; however, different STS structures may enhance the growth of different types of biotic communities and, thus, affect the nutrient uptake capabilities of the stream (Argerich et al., 2011).

\section{Conclusions}

This paper introduces a classification scheme that categorizes different types of STS in riverine systems based on their flow structure. Eight types of STS are identified and, in some cases, subcategorized on the basis of differing characteristic mean flow structure: (1) lateral cavities (emergent and submerged); (2) protruding in-channel flow obstructions (backward- and forward-facing step); (3) isolated in-channel flow obstructions (emergent and submerged); (4) cascades and riffles; (5) aquatic vegetation (emergent and submerged); (6) pools (vertically submerged cavity, closed cavity, and recirculating reservoir); (7) meander bends; and (8) confluence of streams. This classification scheme provides a foundation for studying different types of STS with greater quantitative accuracy so that greater insight will be gained into key hydromorphic parameters influencing mass and momentum exchange.

The long-term goal is to develop predictive mean residence times and RTDs for different types of STS using fieldmeasureable hydromorphic parameters, which will provide the ability to quantitatively separate STS from HTS. In some cases, predictive relationships may need to be developed for ranges of STS geometries and Reynolds numbers. To implement the STS classification scheme, we propose a method that deconvolves STS mean residence times using predictive relationships from the total transient storage RTD (measured from a tracer test) to obtain an estimate of the HTS RTD.

\section{Appendix A}

\section{Notation.}

\begin{tabular}{|c|c|}
\hline$a$ & Frontal area per canopy volume, $\left[\mathrm{L}^{-1}\right]$ \\
\hline$B$ & Channel width, [L] \\
\hline$C_{\mathrm{D}}$ & Canopy drag coefficient, $[-]$ \\
\hline $\begin{array}{l}C_{\mathrm{f}} \\
d\end{array}$ & $\begin{array}{l}\text { Chézy-type friction coefficient, }[-] \\
\text { Stem diameter, }[\mathrm{L}]\end{array}$ \\
\hline$d_{\mathrm{C}}$ & Main channel depth, $[\mathrm{L}]$ \\
\hline$d_{\mathrm{C}}-d_{\mathrm{STS}}$ & Submergence depth, [L] \\
\hline$d_{\mathrm{C}}-H_{\mathrm{g}}$ & $\begin{array}{l}\text { Mean submergence depth of sediment } \\
\text { grains, [L] }\end{array}$ \\
\hline$d_{\mathrm{E}}$ & Mean water depth in mixing layer, [L] \\
\hline$d_{\mathrm{E}, \mathrm{BFS}}$ & $\begin{array}{l}\text { Mean water depth in mixing layer of a } \\
\text { backward-facing step, [L] }\end{array}$ \\
\hline$d_{\mathrm{E}, \mathrm{FFS}}$ & $\begin{array}{l}\text { Mean water depth in mixing layer of a } \\
\text { forward-facing step, }[\mathrm{L}]\end{array}$ \\
\hline$d_{\mathrm{MI}}$ & $\begin{array}{l}\text { Mean water depth in mixing interface } \\
\text { at stream confluence, [L] }\end{array}$ \\
\hline$d_{\mathrm{STS}}$ & STS water depth, $[\mathrm{L}]$ \\
\hline$d_{\mathrm{STS}, \mathrm{BFS}}$ & Mean depth of backward-facing step, [L] \\
\hline$d_{\mathrm{STS}, \mathrm{FFS}}$ & Mean depth of forward-facing step, $[\mathrm{L}]$ \\
\hline$D$ & $\begin{array}{l}\text { Diffusion coefficient (emergent } \\
\text { vegetation), }\left[\mathrm{L}^{2} \mathrm{~T}^{-1}\right]\end{array}$ \\
\hline$D_{\mathrm{g}}$ & Mean grain diameter, $[\mathrm{L}]$ \\
\hline E & $\begin{array}{l}\text { Exchange velocity at STS interface, } \\
{\left[\mathrm{L} \mathrm{T}^{-1}\right]}\end{array}$ \\
\hline$g$ & Gravitational acceleration, $\left[\mathrm{L} \mathrm{T}^{-2}\right]$ \\
\hline$h$ & Submerged canopy height, [L] \\
\hline
\end{tabular}


$H_{\mathrm{g}} \quad$ Sediment grain height, [L]

$k \quad$ Dimensionless entrainment coefficient, [-]

$L \quad$ STS length (parallel to flow), [L]

$L_{\mathrm{R}} \quad$ Cascade/riffle reach length, [L]

$R \quad$ Meander bend radius of curvature, [L]

$R_{\text {min }} \quad$ Minimum meander bend radius of curvature, [L]

$R / B \quad$ Meander bend curvature ratio, [-]

$S \quad$ Channel slope, [-]

$S_{\mathrm{g}} \quad$ Mean spacing between sediment grains, [L]

$\Delta S \quad$ Mean spacing between individual emergent plant stems, [L]

$t \quad$ Time, [T]

$t_{\mathrm{ml}} \quad$ Total mixing layer thickness (submerged canopy), [L]

$T_{\mathrm{e}} \quad$ Timescale of submerged canopy exchange zone, $[\mathrm{T}]$

$T_{\text {wake }}$ Timescale of submerged canopy wake zone, [T]

$u_{*} \quad$ Shear velocity, $\left[\mathrm{L} \mathrm{T}^{-1}\right]$

$U \quad$ Mean main channel velocity, $\left[\mathrm{L} \mathrm{T}^{-1}\right]$

$U_{1} \quad$ Mean main channel velocity at stream confluence, $\left[\mathrm{L} \mathrm{T}^{-1}\right.$ ]

$U_{2} \quad$ Mean tributary channel velocity at stream confluence, $\left[\mathrm{L} \mathrm{T}^{-1}\right.$ ]

$U_{\mathrm{c}} \quad$ Mean canopy velocity, $\left[\mathrm{L} \mathrm{T}^{-1}\right]$

$\Delta U \quad$ Velocity difference between channel and wake region velocity, $\left[\mathrm{L} \mathrm{T}^{-1}\right]$

$V_{\mathrm{STS}} \quad$ Reach volume of surface transient storage zones, $\left[\mathrm{L}^{3}\right]$

$W \quad$ STS width (normal to flow), [L]

$W_{\mathrm{MI}}$, Width of mixing interface (normal to flow) at stream confluence, [L]

$x \quad$ Downstream distance from point of tracer injection, [L]

$x_{\text {BFS }} \quad$ Length (parallel to flow) of backwardfacing step recirculation, [L]

$x_{\text {FFS }} \quad$ Length (parallel to flow) of forwardfacing step recirculation, [L]

$y_{\text {FFS }}$ Width (normal to flow) of forwardfacing step recirculation, [L]

$\beta \quad$ Stream confluence angle

$\delta \quad$ Boundary layer displacement thickness, [L]

$\delta_{\mathrm{e}} \quad$ Mixing layer penetration depth (submerged canopy), [L]

$\tau \quad$ Mean residence time, $[\mathrm{T}]$ $\tau_{\text {HTS }}$ Harmonic mean of STS mean residence times, [T]

$\tau_{\text {STS }}$ Harmonic mean of mean residence times in hyporheic zone, [T]

$\theta \quad$ Porosity of isolated obstacle, [-]

$v \quad$ Kinematic viscosity, $\left[\mathrm{L}^{2} \mathrm{~T}^{-1}\right]$

$\gamma \quad$ Inclination angle (measured from upstream streambank to obstacle)

Acknowledgements. This work was supported by the National Science Foundation, EAR 09-43570. We would like to thank Ben O'Connor, Jud Harvey, Ted Endreny, Ross Woods, and all other anonymous reviewers for their exceptional reviews and suggestions, which have improved the outcome of this manuscript.

Edited by: R. Woods

\section{References}

Abbe, T. B. and Mongomery, D. R.: Large woody debris jams, channel hydraulics and habitat formation in large rivers, Reg. River. Res. Manage., 12, 201-221, 1996.

Abbe, T. B. and Mongomery, D. R.: Patterns and processes of wood debris accumulation in the Queets river basin, Washington, Geomorphology, 51, 81-107, 2003.

Ackerman, J. D. and Okubo, A.: Reduced mixing in a marine macrophytes canopy, Functional Ecol., 7, 305-309, 1993.

Addad, Y., Laurence, D., Talotte, C., and Jacob, M. C.: Large Eddy Simulation of a Forward-Backward Facing Step for Acoustic Source Identification, Int. J. Heat Fluid Flow, 24, 562-571, 2003.

Anderson, E. J. and Phanikumar, M. S.: Surface storage dynamics in large rivers: Comparing three-dimensional particle transport, one-dimensional fractional derivative, and multirate transient storage models, Water Resour. Res., 47, W09511, doi:10.1029/2010WR010228, 2011.

Anderson, J. K., Wondzell, S. M., Gooseff, M. N., and Haggerty, R.: Patterns in stream longitudinal profiles and implications for hyporheic exchange flow at the H.J. Andrews Experimental Forest, Oregon, USA, Hydrol. Process., 19, 2931-2949, 2005.

Andrews, E. D.: Scour and Fill in a Stream Channel: East Fork River, Western Wyoming, USGS Professional Paper 1117, US Government Printing Office, Washington, D.C. USA, 1979.

Argerich, A., Martí, E., Sabater, F., Haggerty, R., and Ribot, M.: Influence of transient storage on stream nutrient uptake based on substrata manipulation, Aquat. Sci., 73, 365-377, doi:10.0007/s00027-011-0184-9, 2011.

Armaly, B. F., Durst, F., Pereira, J. C. F., and Schönung, B.: Experimental and theoretical investigation of backward-facing step, J. Fluid Mech., 127, 473-496, 1983.

Ashworth, P. J.: Bedload Transport and Channel Changes in Gravelbed Rivers, Ph.D. thesis, University of Stirling, Stirling, p. 352, 1987.

Awasthi, M.: High Reynolds Number Turbulent Boundary Layer Flow over Small Forward Facing Steps, M.S. Thesis, Virginia Polytechnic Institute and State University, Blacksburg, Virginia, 2012. 
Bagnold, R. A.: Some aspects of the shape of river meanders, US Geol. Surv. Prof. Pap. 282-E, US Government Printing Office, Washington, D.C., USA, 135-144, 1960.

Baker, D. W., Bledsoe, B. P., and Price, Mueller, J.: Stream nitrate uptake and transient storage over a gradient of geomorphic complexity, north-central Colorado, USA, Hydrol. Process., 26, 3241-3252, 2012.

Bathurst, J. C.: Distribution of boundary shear stress in rivers, in: Adjustments of the Fluvial System, edited by: Rhodes, D. D. and Williams, G. P., Kendall-Hart, Dubuque, Iowa, 95-116, 1979.

Bathurst, J. C., Thorne, C. R., and Hey, R. D.: Secondary flow and shear stress at river bends, J. Hydraul. Div. Am. Soc. Civ. Eng., 105, 1277-1295, 1979.

Battin, T. J., Kaplan, L. A., Newbold, J. D., and Hendricks, S. P.: A mixing model analysis of stream solute dynamics and the contribution of hyporheic zone to ecosystem function, Freshwater Biol., 48, 995-1014, 2003.

Bencala, K. E.: Stream-Groundwater Interactions, in: Treatise on Water Science, edited by: Wilderer, P., Academic Press, Oxford, 537-546, 2011.

Bencala, K. E. and Walters, R. A.: Simulation of solute transport in a mountain pool-and-riffle stream: a transient storage model, Water Resour. Res., 19, 718-724, 1983.

Bencala, K. E., Kennedy, V. C., Zellweger, G. W., Jackman, A. P., and Avanzino, R. J.: Interactions of solutes and streambed sediments, 1, An experiment analysis of cation and anion transport in a mountain stream, Water Resour. Res., 20, 1797-1803, 1984.

Bencala, K. E., Gooseff, M. N., and Kimball, B. A.: Rethinking hyporheic flow and transient storage to advance understanding of stream-catchment connections, Water Resour. Res., 47, W00H03, doi:10.1029/2010WR010066, 2011.

Benner, S. G., Smart, E. W., and Moore, J. N.: Metal behavior during surface-groundwater interaction, Silver Bow Creek, Montana, Environ. Sci. Technol., 29, 1789-1795, 1995.

Bennett, S. J., Pirim, T., and Barkdoll, B. D.: Using simulated emergent vegetation to alter stream flow direction within a straight experimental channel, Geomorphology, 44, 115-126, 2002.

Berkowitz, B. and Scher, H.: Theory of anomalous chemical transport in random fracture networks, Phys. Rev. E, 57, 5858-5869, 1998.

Berkowitz, B., Emmanuel, S., and Scher, H.: Non-Fickian transport and multiple-rate mass transfer in porous media, Water Resour. Res., 44, W03402, doi:03410.01029/02007WR005906, 2008.

Biron, P., Best, J. L., and Roy, A. G.: Effects of bed discordance on flow dynamics at open channel confluences, J. Hydraul. Eng.ASCE, 122, 676-682, 1996a.

Biron, P., Boy, A. G., and Best, J. L.: Turbulent flow structure at concordant and discordant open-channel confluences, Exp. Fluids, 21, 437-446, 1996 b.

Blanckaert, K.: Topographic steering, flow recirculation, velocity redistribution, and bed topography in sharp meander bends, Water Resour. Res., 46, W09506, doi:10.1029/2009WR008303, 2010.

Blanckaert, K. and de Vriend, H. J.: Secondary flow in sharp open-channel bends, J. Fluid Mech., 498, 353-380, doi:10.1017/S0022112003006979, 2004.

Blanckaert, K. and de Vriend, H. J.: Meander dynamics: A nonlinear model without curvature restrictions for flow in open-channel bends, J. Geophys. Res., 115, F04011,
doi:10.1029/2009JF001301, 2010.

Blanckaert, K. and Graf, W.: Mean flow and turbulence in open channel bend, J. Hydraul. Eng., 127, 835-847, doi:10.1061/(ASCE)0733-9429(2001)127:10(835), 2001.

Boano, F., Packman, A. I., Cortis, A., Revelli, R., and Ridolfi, L.: A continuous time random walk approach to the stream transport of solutes, Water Resour. Res., 43, W10425, doi:10.1029/2007WR006062, 2007.

Booker, D. J., Sear, D. A., and Payne, A. J.: Modelling threedimensional flow structures and patterns of boundary shear stress in a natural pool-riffle sequence, Earth Surf. Proc. Land., 26, 553-576, 2001.

Boulton, A. J.: Stream ecology and surface-hyporheic exchange: implications, techniques and limitations, Aust. J. Mar. Freshwater Res., 44, 553-564, 1993.

Boulton, A. J., Findlay, S., Marmonier, R., Stanley, E. H., and Valett, H. M.: The functional significance of the hyporheic zone in streams and rivers, Annu. Rev. Ecol. Syst., 29, 59-81, 1998.

Bradbrook, K. F., Biron, P. M., Lane, S. N., Richards, K. S., and Roy, A. G.: Investigation of controls on secondary circulation in a simple confluence geometry using a threedimensional numerical model, Hydrol. Process., 12, 1371-1396, doi:10.1002/(SICI)1099-1085(19980630)12:8<1371::AIDHYP620>3.0.CO;2-C 1998.

Braza, M., Chassaing, P., and Minh, H. H.: Prediction of LargeScale Transition features in the wake of a Circular Cylinder, Phys. Fluids, A2, 1461-1471, 1990.

Briggs, M. A., Gooseff, M. N., Arp, C. D., and Baker, M. A.: A Method for Estimating Surface Transient Storage Parameters for Streams with Concurrent Hyporheic Exchange, Water Resour. Res., 45, W00D27, doi:10.1029/2008WR006959, 2009.

Brunke, M. and Gonser, T.: The ecological significance of exchange processes between rivers and groundwater: Freshwater Biol., 37, 1-33, 1997.

Burggraf, O. R.: Analytical and numerical studies of the structure of steady separated flows, J. Fluid Mech., 24, 113-115, 1966.

Campbell, A. J. and Sidle, R. C.: Bedload transport in a pool-riffle sequence of a coastal Alaska stream, Water Resour. Bull., 21, 218-223, 1985.

Camussi, R., Felli, M., Pereira, F., Aloisio, G., and Di Marco, A.: Statistical Properties of Wall Pressure Fluctuations over a Forward Facing Step, Phys. Fluids, 294, 177-204, 2008.

Cardenas, M. B., Wilson, J. L., and Haggerty, R.: Residence time of bedform-driven hyporheic exchange, Adv. Water Resour., 31, 1382-1386, doi:1310.1016/j.advwatres.2008.1307.1006, 2008.

Carling, P. A.: An appraisal of the velocity reversal hypothesis for stable pool/riffle sequences in the River Severn, England, Earth Surf. Proc. Land., 16, 19-31, 1991.

Castro, I. P. and Dianat, M.: Surface flow patterns on rectangular bodies in thick boundary Layer, J. Fluid Mech., 11, 107-119, 1983.

Cerling, T. E., Morrison, S. J., and Sobocinski, R. W.: Sedimentwater interaction in a small stream: Adsorption of ${ }^{137} \mathrm{Cs}$ by bed load sediments, Water Resour. Res., 26, 1165-1176, 1990.

Chang, K., Constantinescu, G., and Park, S. O.: Analysis of the flow and mass transfer processes for the incompressible flow past an open cavity with a laminar and a fully turbulent incoming boundary layer, J. Fluid Mech., 561, 113-145, 2006. 
Chang, K., Constantinescu, G., and Park, S. O.: Purging of a neutrally buoyant or a dense miscible contaminant from a rectangular cavity, II: Case of an incoming fully turbulent overflow, J. Hydraul. Eng., 133, 373-385, doi:10.1061/(ASCE)07339429(2007)133:4(373), 2007.

Chen, Y. T., Nie, J. H., Hsieh, H. T., and Sun, L. J.: Threedimensional convection flow adjacent to inclined backwardfacing step, Int. J. Heat Mass Transfer, 49, 4795-4803, 2006.

Cheng, M. and Hung, K. C.: Vortex structure of steady flow in a rectangular cavity, Comput. Fluids, 35, 1046-1062, 2006.

Choi, J., Harvey, J. W., and Conklin, M. H.: Characterizing multiple timescales of stream and storage zone interaction that affect solute fate and transport in streams, Water Resour. Res., 36, 1511-1518, doi:10.1029/2000WR900051, 2000.

Clifford, N. J.: Formation of riffle-pool sequences: field evidence for an autogenetic process, Sediment. Geol., 85, 39-51, 1993.

Constantinescu, G. S., Sukhodolov, A., and McCoy, A.: Mass exchange in a shallow channel flow with a series of groynes: LES study and comparison with laboratory and field experiments, Environ. Fluid Mech., 9, 587-615, doi:10.1007/s10652-009-91552, 2009.

Constantinescu, G. S., Miyawaki, S., Rhoads, B., Sukhodolov, A., and Kirkil, G.: Structure of turbulent flow at a river confluence with momentum and velocity ratios close to 1: Insights from an eddy-resolving numerical simulation, Water Resour. Res., 47, W05507, doi:10.1029/2010WR010018, 2011.

Constantinescu, G. S., Miyawaki, S., and Liao, Q.: Flow and turbulence structure past a cluster of freshwater mussels, J. Hydraul. Eng.-ASCE, 139, 347-358, doi:10.1061/(ASCE)HY.19437900.0000692, 2013.

Danckwerts, P. V.: Continuous-flow systems: Distribution of residence times, Chem. Eng. Sci., 2, 1-13, doi:10.1016/00092509(53)80001-1, 1953.

De Angelis, D. L., Loreau, M., Neergaard, D., Mulholland, P. J., and Marzolf, E. R.: Modelling nutrient-periphyton dynamics in streams: the importance of transient storage zones, Ecol. Model., 80, 149-160, 1995.

D’Angelo, D. J., Webster, J. R., Gregory, S. V., and Meyer, J. L.: Transient storage in Appalachian and Cascade mountain streams as related to hydraulic characteristics, J. N. Am. Benthol. Soc., 12, 223-235, 1993.

De Serres, B., Roy, A. G., Biron, P., and Best, J. L.: Threedimensional structure of flow at a confluence of river channels with discordant beds, Geomorphology, 26, 313-335, 1999.

Devenport, W. J. and Simpson, R. L.: Time-dependent and timeaveraged turbulence structure at a rectangular bridge pier with a low flow angle of attack, Intl. conf. fluvial Hydraul., River Flow 2010, Braunschweig, Germany, 1990.

Dietrich, W. E. and Smith, J. D.: Influence of the point bar on flow through curved channels, Water Resour. Res., 19, 1173-1192, doi:10.1029/WR019i005p01173, 1983.

Durst, F. and Tropea, C.: Turbulent, backward-facing step flows in two-dimensional ducts and channels, in: Proc. Third Intl. Symp. on Turbulent Shear Flows, University of California, Davis, 18.1-18.5, 1981.

Elawady, E., Michiue, M., and Hinokidani, O.: Movable bed scour around submerged spur-dikes, Annu. J. Hydraul. Eng., 45, 373378,2000 .
Endreny, T., Lautz, L., and Seigel, D. I.: Hyporheic flow path response to hydraulic jumps at river steps: Flume and hydrodynamic models, Water Resour. Res., 47, W02517, doi:10.1029/2009WR008631, 2011.

Engelhardt, C., Krüger, A., Sukhodolov, A., and Nicklisch, A.: A study of phytoplankton spatial distributions, flow structure and characteristics of mixing in a river reach with groynes, J. Plankton Res., 26, 1351-1366, 2004.

Ensign, S. H. and Doyle, M. W.: In-channel transient storage and associated nutrient retention: Evidence from experimental manipulations, Limnol. Oceanogr., 50, 1740-1751, 2005.

Farabee, T. M. and Casarella, M. J.: Measurement of fluctuating wall pressure for separated/reattached boundary layer flows, J. Vibr. Acoust. Stress Reliabil. Design, 108, 301-307, 1986.

Ferguson, R. I., Parsons, D. R., Lane, S. N., and Hardy, R. J.: Flow in meander bends with recirculation at the inner bank, Water Resour. Res., 39, 1322, doi:10.1029/2003WR001965, 2003.

Fessler, J. R. and Eaton, J. K.: Turbulence modification by particles in a backward-facing step flow, J. Fluid Mech., 394, 97-117, 1999.

Finnigan, J.: Turbulence in Plant Canopies, Annu. Rev. Fluid Mech., 32, 519-571, 2000.

Fiorentini, E., Felli, M., Pereira, F., Camussi, R., and Di Marco, A.: Wall Pressure Fluctuations Over a Forward-Facing Step, 13th AIAA/CEAS Aeroacoustics Conference, Rome, Italy, 1-10, 2007.

Fitzmaurice, L., Shaw, R., Paw, K. U., and Patton, E.: Threedimensional scalar micro-front systems in a large-eddy simulation of vegetation canopy flow, Bound.-Lay. Meteorol., 112, 107-127, 2004.

Gabriel, M. and Boufadel, M.: Estimation of transient storage parameters from a stream tracer study, Hydrology Days, AWRA conference, Colorado, 2002.

Gambi, M., Nowell, A., and Jumars, P.: Flume observations on flow dynamics in Zostera marina (eelgrass) beds, Mar. Ecol.-Prog. Ser., 61, 159-169, 1990.

Gasset, N., Poitras, G. J., Gagnon, Y., and Brothers, C.: Study of atmospheric boundary layer flow over a coastal cliff, Wind Eng., 29, 3-24, 2005.

Ghia, U., Ghia, K. N., and Shin, C. T.: High-Re solutions for incompressible flow using the Navier-Stokes equations and a multigrid method, J. Comput. Phys., 48, 387-411, 1982.

Ghisalberti, M. and Nepf, H.: Mixing layers and coherent structures in vegetated aquatic flow, J. Geophys. Res., 107, 3011, doi:10.1029/2001JC000871, 2002.

Ghisalberti, M. and Nepf, H.: The limited growth of vegetated shear layers, Water. Resour. Res., 40, W07502, doi:10.1029/2003WR002776, 2004.

Ghisalberti, M. and Nepf, H.: Mass transfer in vegetated shear flows, Environ. Fluid Mech., 5, 527-551, doi:10.1007/s10652005-0419-1, 2005.

Ghisalberti, M. and Nepf, H.: The structure of the shear layer over rigid and flexible canopies, Environ. Fluid Mech., 6, 277-301, doi:10.1007/s10652-006-0002-4, 2006.

Ghisalberti, M. and Nepf, H.: Shallow flows over a permeable medium: the hydrodynamics of submerged aquatic canopies, Transp. Porous Media, 78, 385-402, 2009. 
Gooseff, M. N., Wondzell, S. M., Haggerty, R., and Anderson, J.: Comparing transient storage modeling and residence time distribution (RTD) analysis in geomorphically varied reaches in the Lookout Creek basin, Oregon, USA, Adv. Water Resour., 26, 925-937, 2003.

Gooseff, M. N., Anderson, J. K., Wondzell, S. M., LaNier, J., and Haggerty, R.: A modelling study of hyporheic exchange pattern and the sequence, size, and spacing of stream bedforms in mountain stream networks, Oregon, USA, Hydrol. Process., 19, 29152929, 2005.

Gooseff, M. N., Anderson, J. K., Wondzell, S. M., LaNier, J., and Haggerty, R.: A modelling study of hyporheic exchange pattern and the sequence, size, and spacing of stream bedforms in mountain stream networks, Oregon, USA, Hydrol. Process., 20, 24432457, 2006.

Gooseff, M. N., Hall, R. O., and Tank, J. L.: Relating transient storage to channel complexity in streams of varying land use in Jackson Hole, Wyoming, Water Resour. Res., 43, W01417, doi:10.1029/2005WR004626, 2007.

Gooseff, M. N., Payn, R. A., Zarnetske, J. P., Bowden, W. B., McNamara, J. P., and Bradford, J.H.: Comparison of in-channel mobile-immobile zone exchange during instantaneous and constant rate stream tracer additions: Implications for design and interpretation of non-conservative tracer experiments, J. Hydrol., 357, 112-124, 2008.

Gooseff, M. N., Benson, D. A., Briggs, M. A., Weaver, M., Wollheim, W., Peterson, B., and Hopkinson, C. S.: Residence time distributions in surface transient storage zones in streams: Estimation via signal deconvolution, Water Resour. Res., 47, W05509, doi:10.1029/2010WR009959, 2011.

Gücker, B. and Boëchat, G.: Stream morphology controls ammonium retention in tropical headwaters, Ecology, 5, 2818-2827, 2004.

Hack, J. T.: Studies in Longitudinal Stream Profiles in Virginia and Maryland, USGS Professional Paper 294B, US Government printing office, Washington, D.C., USA, 1957.

Haggerty, R., McKenna, S. A., and Meigs, L. C.: On the late-time behavior of tracer test breakthrough curves, Water Resour. Res., 36, 3467-3479, 2000.

Haggerty, R., Wondzell, S. M., and Johnson, M. A.: Powerlaw residence time distribution in the hyporheic zone of a 2nd-order mountain stream, Geophys. Res. Lett., 29, 1640, doi:10.1029/2002GL014743, 2002.

Haggerty, R., Marté, E., Argerich, A., von Schiller, D., and Grimm, N. B.: Resazurin as a "smart" tracer for quantifying metabolically active transient storage in stream ecosystems, J. Geophys. Res.Biogeo., 114, G03014, doi:10.1029/2008JG000942, 2009.

Hall, R. O., Bernhardt, E. S., and Likens, G. E.: Relating nutrient uptake with transient storage in forested mountain streams, Limnol. Oceanogr., 47, 255-265, 2002.

Harvey, J. W. and Bencala, K. E.: The effect of streambed topography on surface-subsurface water exchange in mountain catchments, Water Resour. Res., 29, 89-98, 1993.

Harvey, J. W. and Fuller, C. C.: Effect of enhanced manganese oxidation in the hyporheic zone on basin-scale geochemical mass balance, Water Resour. Res., 34, 623-636, 1998.
Harvey, J. W. and Wagner, B. J.: Quantifying hydrologic interactions between streams and their subsurface hyporheic zones, in: Streams and Groundwaters, edited by: Jones, J. B. and Mulholland, P. J., Academic Press, San Diego, 9-10, 2000.

Harvey, J. W., Wagner, B. J., and Bencala, K. E.: Evaluating the reliability of the stream tracer approach to characterize streamsubsurface water exchange, Water Resour. Res., 32, 2441-2451, 1996.

Harvey, J. W., Conklin, M. H., and Koelsch, R. S.: Predicting changes in hydrologic retention in an evolving semi-arid alluvial stream, Adv. Water Resour., 26, 939-950, 2003.

Harvey, J. W., Saiers, J., and Newlin, J.: Solute transport and storage mechanisms in wetlands of the Everglades, south Florida, Water Resour. Res., 41, W05009, doi:10.1029/2004WR003507, 2005.

Harvey, J. W., Schaffranek, R. W., Noe, G. B., Larsen, L. G., Nowacki, D. J., and O'Connor, B. L.: Hydroecological factors governing surface-water flow on a low gradient floodplain, Water Resour. Res., 45, W03421, doi:10.1029/2008WR007129, 2009.

Hey, R. D. and Thorne, C.R., Secondary flows in river channels, Area, 7, 191-195, 1975.

Hinterberger, C., Fröhlich, J., and Rodi, W.: Three-Dimensional and Depth-Averaged Large-Eddy Simulations of Some Shallow Water Flows, J. Hydraul. Eng.-ASCE, 133, 857-872, 2007.

Hirsch, P. J. and Abrahams, A. D.: The properties of bed sediments in pools and riffles, J. Sediment. Res., 51, 757-760, 1981.

Hodskinson, A. and Ferguson, R. I.: Numerical modelling of separated flow in river bends: Model testing and experimental investigation of geometric controls on the extent of flow separation at the concave bank, Hydrol. Process., 12, 1323-1338, 1998.

Hung, L., Moin, P., and Kim, J.: Direct numerical simulation of turbulent flow over a turbulent-facing step, J. Fluid Mech., 330, 349-374, 1997.

Ikeda, S. and Kanazawa, M.: Three-dimensional organized vortices above flexible water plants, J. Hydraul. Eng.-ASCE, 122, 634 640, 1996.

Iseya, F. and Ikeda, H.: Pulsations in bedload transport rates induced by a longitudinal sediment sorting: A flume study using sand and gravel mixtures, Geograf. Ann. A, 69, 15-27, 1987.

Jackson, T. R., Haggerty, R., Apte, S. V., Coleman, A., and Drost, K. J.: Defining and measuring the mean residence time of lateral surface transient storage zones in small streams, Water Resour. Res., 48, W10501, doi:10.1029/2012WR012096, 2012.

Jackson, T. R., Haggerty, R., and Apte, S. V.: A Predictive Relationship for the Mean Residence Time of Lateral Cavities in GravelBed Rivers and Streams: Incorporating Streambed Roughness and Cavity Shape, Water Resour. Res., doi:10.1002/wrcr.20272, in press, 2013.

Jamieson, C., Post, G., and Rennie, C. D.: Spatial variability of three-dimensional Reynolds stresses in a developing channel bend, Earth Surf. Proc. Land., 35, 1029-1043, doi:10.1002/esp.1930, 2010.

Johannesson, H. and Parker, G.: Secondary flow in mildly sinuous channel, J. Hydraul. Eng.-ASCE, 115, 289-308, doi:10.1061/(ASCE)0733-9429(1989)115:3(289), 1989.

Jouon, A., Douillet, P., Ouillon, S., and Fraunié, P.: Calculations of hydrodynamic time parameters in a semi-opened coastal zone using a 3D hydrodynamic model, Cont. Shelf Res., 26, 1395$1415,2006$. 
Kang, S. and Sotiropoulos, F.: Flow phenomena and mechanisms in a field-scale experimental meandering channel with a pool-riffle sequence: Insights gained via numerical simulation Flow phenomena and mechanisms in a field-scale experimental meandering channel with a pool-riffle sequence: Insights gained via numerical simulation, J. Geophys. Res., 116, F03011, doi:10.1029/2010JF001814, 2011.

Kasahara, T. and Wondzell, S. M.: Geomorphic controls on hyporheic exchange flow in mountain streams, Water Resour. Res., 39, 1005, doi:10.1029/2002WR001386, 2003.

Keller, E. A.: Form and Fluvial Processes of Dry Creek, near Winters, California, M.S. Thesis, University of California, Davis, 1969.

Keller, E. A.: Areal sorting of bed material: the hypothesis of velocity reversal, Geol. Soc. Am. Bull., 83, 915-918, 1971.

Keller, E. A. and Florsheim, J. L.: Velocity reversal hypothesis: A model approach, Earth Surf. Proc. Land., 18, 733-740, 1993.

Kim, J., Kline, S. J., and Johnston, J. P.: Investigation of a reattaching turbulent shear layer: Flow over a backward-facing step, Proceedings of the Winter Annual Meeting, December 1979, New York, N.Y., 41-48, 1979.

Kirkil, G. and Constantinescu, S. G.: Nature of flow and turbulence structure around an in-stream vertical plate in a shallow channel and the implications for sediment erosion, Water Resour. Res., 45, W06412, doi:10.1029/2008WR007363, 2009.

Kirkil, G., Constantinescu, S. G., and Ettema, R.: Investigation of the velocity and pressure fluctuation distributions inside the turbulent horseshoe vortex system around circular bridge pier, Intl conf. fluvial Hydraul., River Flow 2006, September 2006, Lisbon, Portugal, 2006.

Kirkil, G., Constantinescu, S. G., and Ettema, R.: Coherent structures in the flow field around a circular cylinder with scour hole, J. Hydraul. Eng.-ASCE, 134, 572-587, 2008.

Kiya, M. and Sasaki, K.: Structure of a turbulent separation bubble, J. Fluid Mech., 137, 83-113, 1983.

Kuehn, D. M.: Some effects of adverse pressure gradient on the incompressible reattaching flow over a rearward-facing step, AIAA J., 18, 343-344, 1980.

Kurzke, M., Weitbrecht, V., and Jirka, G. H.: Laboratory concentration measurements for determination of mass exchange between groin fields and main stream, paper presented at IAHR Conference, "River Flow", Louvain de la Neuve, Belgium, 2002.

Laenen, A. and Bencala, K. E.: Transient Storage Assessments of Dye-Tracer Injections in Rivers of the Willamette Basin, Oregon, J. Am. Water Resour. As., 37, 367-377, 2001.

Lamb, M. P., Howard, A. D., Dietrich, W. E., and Perron, J. T.: Formation of amphitheater-headed valleys by waterfall erosion after large-scale slumping on Hawai'i, Geol. Soc. Am. Bull., 119, 805-822, 2007.

Lancaster, J. and Hildrew, A. G.: Characterizing in-stream flow refugia, Can. J. Fish. Aquat. Sci., 50, 1663-1675, 1993.

Lane, E. W. and Borland, W .M.: River-bed scour during floods, Trans. Am. Soc. Civil Eng., 119, 1069-1079, 1954.

Langmuir, I.: The velocity of reactions in gases moving through heated vessels and the effect of convection and diffusion, J. Am. Chem. Soc., 30, 1742-1754, 1908.

Lautz, L. K. and Siegel, D. I.: Modeling surface and ground water mixing in the hyporheic zone using MODFLOW and MT3D, Adv. Water Resour., 29, 1618-1633, 2006.
Lautz, L. K. and Siegel, D. I.: The effect of transient storage on nitrate uptake lengths in streams: An inter-site comparison, Hydrol. Process., 21, 3533-3548, 2007.

Lautz, L. K., Siegel, D. I., and Bauer, R. L.: Impact of debris dams on hyporheic interaction along a semi-arid stream, Hydrol. Process., 20, 183-196, 2006.

Lawson, S. J. and Barakos, G. N., Review of numerical simulations for high-speed, turbulent cavity flows, Aerosp. Sci. Technol., 47, 186-216, 2011.

Leclercq, D. J., Jacob, M. C., Louisot, A., and Talotte, C.: ForwardBackward facing step pair: Aerodynamic flow, wall pressure and acoustic characterization, Proc. 7th AIAA/CEAS Aeroacoustics Conference, Seoul, Korea, 075113-1-075113-13, 2009.

Leeder, M. R. and Bridges, P. H.: Flow separation in meander bends, Nature, 253, 338-339, doi:10.1038/253338a0, 1975.

Leopold, L. B., Bagnold, R. A., Wolman, M. G., and Brush, L. M.: Flow resistance in sinuous and irregular channels, US Geol. Surv. Prof. Pap. 282D, US Government printing office, Washington, D.C., USA, 111-134, 1960.

Lightbody, A. and Nepf, H.: Prediction of velocity profiles and longitudinal dispersion in emergent salt marsh vegetation, Limnol. Oceanogr., 51, 218-228, 2006.

Lin, J. C. and Rockwell, D.: Organized oscillations of initially turbulent flow past a cavity, AIAA J., 39, 1139-1151, 2001.

Lisle, T. E.: A sorting mechanism for a riffle-pool sequence, Geol. Soc. Am. Bull., 90, 1142-1157, 1979.

Lisle, T. E. and Hilton, S.: The volume of fine sediment in pools: an index of sediment supply in gravel-bed streams, Water Resour. Bull., 28, 371-383, 1992.

Lisle, T. E., Ikeda, H., and Iseya, F.: Formation of stationary alternate bars in a steep channel with mixed-size sediment: A flume experiment, Earth Surf. Proc. Land., 16, 463-469, 1991.

McClain, M. E., Boyer, E. W., Dent, C. L., Gergel, S. E., Grimm, N. B., Groffman, P. M., Hart, S. C., Harvey, J. W., Johnston, C. A., Mayorga, E., McDowell, W. H., and Pinay, G.: Biogeochemical hot spots and hot moments at the interface of terrestrial and aquatic Ecosystems, Ecosystems, 6, 301-312, 2003.

McCoy, A., Constantinescu, G., and Weber, L.: Exchange processes in a channel with two vertical emerged obstructions, Flow Turbul. Combust., 77, 97-126, doi:10.1007/s10494-006-9039-1, 2006.

McCoy, A., Constantinescu, G., and Weber, L.: A numerical investigation of coherent structures and mass exchange processes in channel flow with two lateral submerged groynes, Water Resour. Res., 43, W05445, doi:10.1029/2006WR005267, 2007.

McCoy, A., Constantinescu, G., and Weber, L.: Numerical investigation of flow hydrodynamics in a channel with a series of groynes, J. Hydraul. Eng.-ASCE, 134, 157-172, 2008.

Miyawaki, S., Constantinescu, S. G., Kirkil, G., Rhoads, B., and Sukhodolov, A.: Numerical investigation of three-dimensional flow structure at a river confluence, Proceedings of the 33rd IAHR Congress: Water Engineering for a Sustainable Environment, Vancouver, Canada, 2009.

Miyawaki, S., Constantinescu, S. G., Rhoads, B., and Sukhodolov, A.: On the three-dimensional flow structure at a river confluence with a high momentum ratio, Intl. conf. on Fluvial Hydraulics, River Flow 2010, September 2010, Braunschweig, Germany, 2010. 
Montgomery, D. R. and Buffington, J. M.: Channel reach morphology in mountain drainage basins, Geol. Soc. Am. Bull., 109, 596-611, 1997.

Montroll, E. W. and Weiss, G. H.: Random walks on Lattices, II, J. Math. Phys., 6, 167-181, 1965.

Morrice, J. A., Valett, H. M., Dahm, C. N., and Campana, M. E.: Alluvial characteristics, groundwater-surface water exchange and hydrologic retention in headwater streams, Hydrol. Process., 11, 253-267, 1997.

Moss, W. D. and Baker, S.: Recirculating Flows Associated With Two Dimensional Steps, Aeronaut. Quarter., 151-172, 1980.

Mulholland, P. J., Marzolfl, E. R., Webster, J. R., Hart, D. R., and Hendricks, S. P.: Evidence that hyporheic zones increase heterotrophic metabolism and phosphorus uptake in forest streams, Limnol. Oceanogr., 42, 443-451, 1997.

Nepf, H.: Drag, turbulence, and diffusion in flow through emergent vegetation, Water Resour. Res., 35, 479-489, 1999.

Nepf, H.: Flow and transport in regions with aquatic vegetation, Annu. Rev. Fluid Mech., 44, 123-142, 2012.

Nepf, H. and Ghisalberti, M.: Flow and transport in channels with submerged vegetation, Acta Geophys., 56, 753-777, 2008.

Nepf, H. and Koch, E.: Vertical secondary flows in submersed plantlike arrays, Limnol. Oceanogr., 44, 1072-1080, 1999.

Nepf, H. and Vivoni, E. R.: Flow structure in depth-limited, vegetated flow, J. Geophys. Res., 105, 28547-28557, 2000.

Nepf, H., Ghisalberti, M., White, B., and Murphy, E.: Retention time and dispersion associated with submerged aquatic canopies, Water Resour. Res., 43, W04422, doi:10.1029/2006WR005362, 2007.

Newbold, J. D., Elwood, J. W., O'Neill, R. V., and Sheldon, A. L.: Phosphorus dynamics in a woodland stream ecosystem: A study of nutrient spiraling, Ecology, 64, 1249-1265, 1983.

Nikora, V., Goring, D., McEwan, I., and Griffiths, G.: Spatially averaged open channel flow over rough bed, J. Hydraul. Eng.-ASCE, 127, 123-133, 2001.

Nikora, V. I., McEwan, I. K., McLean, S. R., Coleman, S. E., Pokrajac, D., and Walters, R.: Double-averaging concept for roughbed open-channel and overland flows: Theoretical background, J. Hydraul. Eng.-ASCE, 133, 873-883, 2007.

O'Connor, B. L., Hondzo, M., and Harvey, J. W.: Predictive modeling of transient storage and nutrient uptake: Implications for stream restoration, J. Hydraul. Eng.-ASCE, 136, 1018-1032, 2010.

Pearson, D. S., Goulart, P. J., and Ganapathisubramani, B.: Investigation of turbulent separation in a forward-facing step flow, J. Phys. Conf. Ser., 318, 022031, doi:10.1088/17426596/318/2/022031, 2001.

Peng, J. and Kawahara, Y.: Numerical simulation of flow in river with spur dikes, in: Collected Papers of River and Environmental Engineering Laboratory, report, Univ. of Tokyo, Tokyo, 88-89, 1997.

Peng, J., Kawahara, Y., and Huang, G.: Evaluation of modified k-e models in simulating 3d flows over submerged spur dikes, paper presented at Turbulence and Shear Flow-1, First International Symposium, Am. Soc. Civ. Eng., Santa Barbara, California, 1999.

Peterson, D. F. and Mohanty, P. K.: Flume studies of flow in steep, rough channels, J. Hydraul. Div.-ASCE, 86, 55-79, 1960.
Poggi, D., Porporato, A., Ridolfi, L., Albertson, J. D., and Katul, G. G.: The effect of vegetation density on canopy sub-layer turbulence, Bound.-Lay. Meteorol., 111, 565-587, 2004.

Rai, M. M. and Moin, P.: Direct Numerical Simulation of Transition and Turbulence in a Spatially Evolving Boundary Layer, J. Comp. Phys., 109, 169-192, 1993.

Rathburn, S. and Wohl, E.: Predicting fine sediment dynamics along a pool-riffle mountain channel, Geomorphology, 55, 11-124, 2003.

Raven, P. J., Holmes, N. T. H., Dawson, F. H., Kox, P. J. A., Everard, M., Fozzard, I. R., and Rouen, K. J.: River habitat quality: the physical character of rivers and streams in the UK and Isle of Man, Report No. 2 to the Environment Agency, 1998.

Rhoads, B. L. and Kenworthy, S. T.: Flow structure at an asymmetrical stream confluence, Geomorphology, 11, 273-293, 1995.

Rhoads, B. L. and Kenworthy, S. T.: Time-averaged flow structure in the central region of a stream confluence, Earth Surf. Proc. Land., 23, 171-191, 1998.

Rhoads, B. L. and Sukhodolov, A.: Field investigation of threedimensional flow structure at stream confluences: Part I. Thermal mixing and time-averaged velocities, Water Resour. Res., 37, 2393-2410, 2001.

Rockwell, D.: Prediction of Oscillation Frequencies due to Unstable Flow Past Cavities, J. Fluids Eng., 99, 294-300, 1977.

Rockwell, D.: Invited Lecture: Oscillations of Impinging Shear Layers, AIAA J., 21, 645-664, 1983.

Rockwell, D.: Vortex-Body Interactions, Ann. Rev. Fluid Mech., 30, 199-229, 1998.

Rockwell, D. and Knisely, C.: Unsteady Features of Flow Past a Cavity, J. Hydraul. Div.-ASCE, 105, 969-979, 1979.

Rockwell, D. and Knisely, C.: Observation of the three-dimensional nature of unstable flow past a cavity, Phys. Fluids, 23, 425-431, 1980.

Rockwell, D. and Naudascher, E.: Review self-sustained oscillations of flow past cavities, J. Fluids Eng., 100, 152-165, 1978.

Rozovskii, I. L: Flow of Water in Bends of Open Channels, Acad. of Sci. of the Ukr. SSR, Kiev, 1957.

Rubin, D. M., Schmidt, J. C., and Moore, J. N.: Origin, structure, and evolution of a reattachment bar, Colorado River, Grand Canyon, Arizona, J. Sediment. Res., 60, 982-991, 1990.

Runkel, R. L.: One-Dimensional Transport with Inflow and Storage (OTIS): A Solute Transport Model for Streams and Rivers, US Geol. Surv. Water Resour. Invest. Rep. 98-4018, US Government Printing Office, Washington, D.C., USA, 1998.

Sadeque, M. A. F., Rajaratnam, N., and Loewen, M. R.: Flow around Cylinders in Open Channels, J. Hydraul. Eng.-ASCE, 134, 60-71, 2008.

Salehin, M., Packman, A. I., and Wörman, A.: Comparison of transient storage in vegetated and unvegetated reaches of a small agricultural stream in Sweden: seasonal variation and anthropogenic manipulation, Adv. Water Resour., 26, 951-965, 2003.

Sardin, M., Schweich, D., Keij, F. J., and van Genuchten, M. T.: Modeling the nonequilibrium transport of linearly interacting solutes in porous media: A review, Water Resour. Res., 27, 22872307, doi:10.1029/91WR01034, 1991.

Sarohia, V.: Experimental investigation of oscillations in flows over shallow cavities, AIAA J., 15, 984-991, 1977. 
Schmidt, J. C.: Recirculating flow and sedimentation in the Colorado River in Grand Canyon, Arizona, J. Geol., 98, 709-724, 1990.

Sear, D. A.: Sediment transport processes in pool-riffle sequences, Earth Surf. Proc. Land., 21, 241-262, 1996.

Seo, I. W. and Maxwell, W. H. C.: Modeling low-flow mixing through pools and riffles, J. Hydraul. Eng.-ASCE, 118, 14061423, 1992.

Shen, C. and Floryan, J. M.: Low Reynolds number flow over cavities, Phys. Fluids, 28, 3191-3202, 1985.

Shen, Y. and Diplas, P.: Application of two- and threedimensional computational fluid dynamics models to complex ecological stream flows, J. Hydrol., 348, 195-214, doi:10.1016/j.jhydrol.2007.09.060, 2008.

Sidle, R. C.: Bedload transport regime of a small forest stream, Water Resour. Res., 24, 207-218, 1988.

Silveira Neto, A., Grand, D., Metais, O., and Lesieur, M.: A numerical investigation of the coherent vortices in turbulence behind a backward-facing step, J. Fluid Mech., 256, 1-25, 1993.

Socolofsky, S. A. and Jirka, G. H.: Environmental Fluid Mechanics 1: Mixing and Transport Processes in the Environment, Texas A\&M University Press, Texas, 184 pp., 2005,

Squillace, P. J., Thurman, E. M., and Furlong, E. T.: Groundwater as a nonpoint source of atrazine and deethylatrazine in a river during base flow conditions, Water Resour. Res., 29, 1719-1729, 1993.

Stanford, J. A. and Ward, J. V.: The hyporheic habitat of river ecosystems, Nature, 335, 64-66, 1988.

Stoesser, T., Palau Salvador, G., Rodi, W., and Diplas, P.: Large Eddy Simulation of turbulent flow through submerged vegetation, Transp. Porous Media, 78, 347-365, doi:10.1007/s11242009-9371-8, 2009.

Stofleth, J. M., Shields Jr., F. D., and Fox, G. A.: Hyporheic and total transient storage in small, sand-bed streams, Hydrol. Process., 22, 1885-1894, 2008.

Stonedahl, S. H., Harvey, J. W., Detty, J., Aubeneau, A., and Packman, A. I.: Physical controls and predictability of stream hyporheic flow evaluated with a multiscale model, Water Resour. Res., 48, W10513, doi:10.1029/2011WR011582, 2012.

Sukhodolov, A. and Rhoads, B.: Field investigation of threedimensional flow structure at stream confluences: Part II. Turbulence, Water Resour. Res., 37, 2411-2424, 2001.

Sukhodolov, A. and Sukhodolova, T.: Evolution of mixing layers in turbulent flow over submerged vegetation: field experiments and measurement study, in: River Flow 2006, Two Volume Set, Proceedings of the International Conference on Fluvial Hydraulics, 6-8 September 2006, Lisbon, Portugal, edited by: Alves, E. C. T. L., Cardoso, A. H., Leal, J. G. A. B;, and Ferreira, R. M. L., Taylor \& Francis, 525-534, doi:10.1201/9781439833865.ch54, 2006.

Tanino, Y. and Nepf, H.: Lateral dispersion in random cylinder arrays at high Reynolds number, J. Fluid Mech., 600, 339-371, 2008.

Thomas, S. A., Valett, H. M., Mulholland, P. J., Fellows, C. S., Webster, J. R., Dahm, C. N., and Peterson, C. G.: Nitrogen retention in headwater streams: the influence of groundwater-surface water exchange: Optimizing Nitrogen Management in Food and Energy Production and Environmental Protection: Proceedings of the 2nd International Nitrogen Conference on Science and Policy
TheScientificWorld 2001, 623-631, doi:10.1100/tsw.2001.272, 2001.

Thompson, A.: Secondary flows and the pool-riffle unit: A case study of the processes of meander development, Earth Surf. Proc. Land., 11, 631-641, doi:10.1002/esp.3290110606, 1986.

Thompson, D. M.: Hydraulics and pool geometry, PhD dissertation, Colorado State University, Ft. Collins, 1997.

Thompson, D. M. and Hoffman, K. S.: Pool dimensions in coarsegrained, New England channels, Geol. Soc. Am. Annual Meeting, Abstracts with Programs, 31, A-49, 111th Annual Meeting of The Geological Society of America held 24-28 October 1999, at the Colorado Convention Center (CCC) in Denver, Colorado, 1999.

Thompson, D. M., Wohl, E. E., and Jarrett, R. D.: A revised velocity-reversal and sediment-sorting model for a high-gradient, pool-riffle stream, Phys. Geogr., 17, 142-156, 1996.

Thompson, D. M., Nelson, J. M., and Wohl, E. E.: Interactions between pool geometry and hydraulics, Water Resour. Res., 34, 3673-3681, 1998.

Thomson, J.: On the origin of windings of rivers in alluvial plains, with remarks on the flow of water round bends in pipes, P. Roy. Soc. Lond., 25, 5-8, 1876.

Thorne, C. R., Zevenbergen, L. W., Pitlick, J. C., Rais, S., Bradley, J. B., and Julien, P. Y.: Direct measurements of secondary currents in a meandering sand-bed river, Nature, 315, 746-747, doi:10.1038/315746a0, 1985.

Tominaga, A., Ijima, K., and Nakano, Y.: PIV analysis of flow structures around skewed spur dikes, Annu. J. Hydraul. Eng., 45, 379381, 2001.

Tritico, H. M. and Hotchkiss, R. H.: Unobstructed and Obstructed Turbulent Flow in Gravel Bed Rivers, J. Hydraul. Eng.-ASCE, 131, 635-645, 2005.

Tritton, D. J.: Physical Fluid Dynamics, 2nd Edn., Clarendon, Oxford, 1988.

Uijttewaal, W. S. J., Lehmann, D., and Mazijk, A. V.: Exchange processes between a river and its groyne fields: model experiments, J. Hydraul. Eng.-ASCE, 127, 928-936, 2001.

Uijttewaal, W. S. J.: Effects of groyne layout on the flow in groyne fields: Laboratory experiments, J. Hydraul. Eng.-ASCE, 131, 782-794, 2005.

Valentine, E. M. and Wood, I. R.: Longitudinal dispersion with dead zones, J. Hydraul. Eng.-ASCE, 103, 975-990, 1977.

Valett, H. M., Morrice, J. A., Dahm, C. N., and Campana, M. E.: Parent lithology, surface-groundwater exchange, and nitrate retention in headwater streams, Limnol. Oceanogr., 41, 333-345, 1996.

Valett, H. M., Dahm, C. N., Campana, M. E., Morrice, J. A., Baker, M. A., and Fellows, C. S.: Hydrologic influences on groundwater-surface water ecotones: heterogeneity in nutrient composition and retention, J. N. Am. Benthol. Soc., 16, 239-247, 1997.

van Balen, W., Uijttewaal, W. S. J., and Blanckaert, K.: Large-eddy simulation of a mildly curved open-channel flow, J. Fluid Mech., 630, 413-442, doi:10.1017/S0022112009007277, 2009.

van Bendegom, L.: Some considerations on river morphology and river improvement (in Dutch), De Ingenieur, 59, 1-11, (English translation: Tech. Transl. 1054, Natl. Res. Counc. Can., Ottawa, 1963), 1947. 
Vanoni, V. A.: Sedimentation Engineering, Am. Soc. Civ. Eng., New York, 1975.

Versteeg, H. and Malalasekera, W.: An Introduction to Computational Fluid Dynamics: The Finite Volume Method, 2nd Edn., Prentice Hall, 2007.

Wallast, I., Uijttewaal, W., and van Mazijk, A.: Exchange processes between groyne field and main stream, P. 28th IAHR Congress, Graz, Austria, 1999.

Weitbrecht, V. and Jirka, G. H.: Flow patterns and exchange processes in dead zones of rivers, paper presented at Proc. 29th IAHR Congress, Beijing, China, 2001a.

Weitbrecht, V. and Jirka, G. H.: Flow Patterns In Dead Zones of Rivers and their Effect On Exchange Processes, paper presented at Proc. IAHR 3rd. Int. Symp. Environ. Hydr., Tempe, Arizona, 2001b.

Weitbrecht, V., Socolofsky, S. A., and Jirka, G. H.: Experiments on mass exchange between groin fields and main stream in rivers, $\mathrm{J}$. Hydraul. Eng.-ASCE, 134, 173-183, 2008.

Williams, P. T. and Baker, A. J.: Numerical Simulations of Laminar Flow over a 3-D Backward-Facing Step, Int. J. Num. Methods Fluids, 24, 1159-1183, 1997.

Wilson, C., Stoesser, T., Bates, P., and Pinzen, A.: Open Channel Flow through Different Forms of Submerged Flexible Vegetation. J. Hydraul. Eng.-ASCE, 129, 847-853, 2003.

Wondzell, S., LaNier, J., and Haggerty, R.: Evaluation of alternative groundwater flow models for simulating hyporheic exchange in a small mountain stream, J. Hydrol., 364, 142-151, 2009a.
Wondzell, S., LaNier, J., Haggerty, R., Woodsmith, R., and Edwards, R. T.: Changes in hyporheic exchange flow following experimental wood removal in a small, low gradient Stream, Water Resour. Res., 45, W05406, doi:10.1029/2008WR007214, 2009b.

Wörman, A., Packman, A. I., Johansson, H., and Jonsson, K.: Effect of flow-induced exchange in hyporheic zones on longitudinal transport of solutes in streams and rivers, Water Resour. Res., 38, 1001, doi:1010.1029/2001WR000769, 2002.

Wörman, A., Packman, A. I., Marklund, L., Harvey, J. W., and Stone, S. H.: Fractal topography and subsurface water flows from fluvial bedforms to the continental shield, Geophys. Res. Lett., 34, L07402, doi:10.1029/2007GL029426, 2007.

Wright, S. A. and Kaplinski, M.: Flow structures and sandbar dynamics in a canyon river during a controlled flood, Colorado River, Arizona, J. Geophys. Res., 116, F01019, doi:10.1029/2009JF001442, 2011.

Yossef, M. F. M. and de Vriend, H. J.: Flow Details near River Groynes: Experimental Investigation, J. Hydraul. Eng.-ASCE, 137, 504-516, 2011.

Zarnetske, J. P., Gooseff, M. N., Brosten, T. R., Bradford, J. H., McNamara, J. P., and Bowdens, W. B.: Transient storage as a function of geomorphology, discharge, and permafrost active layer conditions in Arctic tundra streams, Water Resour. Res., 43, 1$13,2007$. 